\title{
OPEN The weak evidence of lip print analysis for sexual dimorphism in forensic dentistry: a systematic literature review and meta-analysis
}

\author{
Ademir Franco ${ }^{1,2,3}$, Lorenna Keren Gomes Lima ${ }^{4}$, Murilo Navarro de Oliveira ${ }^{5}$, \\ Walbert de Andrade Vieira ${ }^{6}$, Cauane Blumenberg ${ }^{7}$, Márcio Magno Costa $^{8}$ \& \\ Luiz Renato Paranhos ${ }^{9 凶}$
}

This study aimed to assess the prevalence of lip print patterns among males and females, and to test the diagnostic accuracy of lip pattern analysis for sexual dimorphism in forensic dentistry. A systematic literature review was performed following the PRISMA guidelines. The search was performed in six primary databases and three databases to cover part of the grey literature. Observational and diagnostic accuracy studies that investigated lip print patterns through cheiloscopy for sexual dimorphism were selected. Risk of bias was assessed with the Joanna Briggs Institute (JBI) tool. Proportion meta-analysis using random effects was fitted to pool the accuracy of cheiloscopy. The odds of correctly identifying males and females was assessed through a random effects metaanalysis. GRADE approach was used to assess certainty of evidence. The search found 3,977 records, published between 1982 and 2019. Seventy-two studies fulfilled the eligibility criteria and were included in the qualitative analysis ( $n=22,965$ participants), and twenty-two studies were sampled for meta-analysis. Fifty studies had low risk of bias. Suzuki and Tsuchihashi's technique was the most prevalent among studies. The accuracy of sexual dimorphism through cheiloscopy ranged between 52.7 and $93.5 \%$, while the pooled accuracy was $76.8 \%(95 \% \mathrm{Cl}=65.8 ; 87.7)$. There was no difference between the accuracy to identify males or females $(\mathrm{OR}=0.71 ; 95 \% \mathrm{Cl}=0.26 ; 1.99)$. The large spectrum of studies on sexual dimorphism via cheiloscopy depicted accuracy percentage rates that rise uncertainty and concern. The unclear performance of the technique could lead to wrong forensic practice.

Cheiloscopy is a field of forensic odontology dedicated to the technical analysis of the human lips ${ }^{1}$. Dating from the 30's, this procedure is carried out in the context of human identification ${ }^{2}$. More specifically, furrows on the vermillion of the lips are assessed based on their alleged distinctive pattern ${ }^{3}$. In practice, there is speculation about the uniqueness of lip print patterns ${ }^{4}$, ethnical variability ${ }^{5}$ and sexual dimorphism ${ }^{6}$.

Human identification methods must rely on scientifically acceptable tools ${ }^{7}$, such as fingerprint, dental and genetic analyses ${ }^{8}$. Authors of cheiloscopy studies suggest that the analysis of lip prints can support the identification process by narrowing down potential victims based on $\mathrm{sex}^{9}$. The contemporary scientific literature on cheiloscopy is vast and growing over time ${ }^{10-15}$. One of the "so-called" advantages of lip prints relies on the alleged unique patterns of furrows that will not repeat between different persons ${ }^{9}$. Authors also claim that lip prints can be found in crime scenes, especially on cigarettes, napkins and glasses ${ }^{9}$. Additionally, the literature points out

\footnotetext{
${ }^{1}$ Centre of Forensic and Legal Medicine and Dentistry, School of Dentistry, University of Dundee, Dundee, Scotland. ${ }^{2}$ Division of Forensic Dentistry, Faculdade São Leopoldo Mandic, Campinas, Brazil. ${ }^{3}$ Department of Therapeutic Stomatology, Institute of Dentistry, Sechenov University, Moscow, Russia. ${ }^{4}$ School of Dentistry, Federal University of Uberlândia, Uberlândia, Brazil. ${ }^{5}$ School of Dentistry, Post-Graduate Program in Dentistry, Federal University of Uberlândia, Uberlândia, Brazil. 'Department of Restorative Dentistry, Endodontics Division, Piracicaba Dental School, State University of Campinas (UNICAMP), Piracicaba, Brazil. ${ }^{7}$ Post-Graduate Program in Epidemiology, School of Social Medicine, Federal University of Pelotas, Pelotas, Brazil. ${ }^{8}$ Division of Removable Prosthesis and Dental Materials, School of Dentistry, Federal University of Uberlândia, Uberlândia, Brazil. ${ }^{9}$ Division of Preventive and Community Dentistry, School of Dentistry, Federal University of Uberlândia, Campus Umuarama, Av. Pará, 1720, Bloco 2G, sala 1, Uberlândia, MG 38405-320, Brazil. ${ }^{\circledR}$ email: paranhos.Irp@gmail.com
} 


\begin{tabular}{|c|c|}
\hline Database & Search Strategy (August, 2020) \\
\hline $\begin{array}{l}\text { PubMed } \\
\text { http://www.ncbi.nlm.nih.gov/pubmed }\end{array}$ & $\begin{array}{l}\text { ((“Cheiloscopy” OR “Lip Print” OR “Lip Pattern” OR “Lipstick”) AND (“Sex” OR “Gender” OR } \\
\text { “Dimorphism”)) }\end{array}$ \\
\hline $\begin{array}{l}\text { Scopus } \\
\text { http://www.scopus.com/ }\end{array}$ & $\begin{array}{l}\text { ((“Cheiloscopy” OR “Lip Print” OR “Lip Pattern” OR “Lipstick”) AND (“Sex” OR “Gender” OR } \\
\text { “Dimorphism”)) }\end{array}$ \\
\hline $\begin{array}{l}\text { LILACS } \\
\text { http://lilacs.bvsalud.org/ }\end{array}$ & $\begin{array}{l}\text { ((“cheiloscopy" OR "lip print” OR “lip pattern” OR “lipstick”) AND (“sex" OR "gender” OR “dimor- } \\
\text { phism”)) AND (instance:"regional") AND ( db:("LILACS")) }\end{array}$ \\
\hline $\begin{array}{l}\text { SciELO } \\
\text { http://www.scielo.org/ }\end{array}$ & ((Cheiloscopy OR Lip Print OR Lip Pattern OR Lipstick) AND (Sex OR Gender OR Dimorphism)) \\
\hline $\begin{array}{l}\text { Embase } \\
\text { http://www.embase.com }\end{array}$ & $\begin{array}{l}\text { ('Cheiloscopy'/exp OR 'cheiloscopy' OR 'lip print'/exp OR 'lip print' OR 'lip pattern' OR 'lipstick'/ } \\
\text { exp OR 'lipstick') AND ('sex'/exp OR 'sex' OR 'gender'/exp OR 'gender' OR 'dimorphism'/exp OR } \\
\text { 'dimorphism') }\end{array}$ \\
\hline $\begin{array}{l}\text { Web Of Science } \\
\text { http://apps.webofknowledge.com/ }\end{array}$ & $\begin{array}{l}\text { ((“Cheiloscopy” OR “Lip Print” OR “Lip Pattern” OR “Lipstick”) AND (“Sex” OR “Gender” OR } \\
\text { “Dimorphism”)) }\end{array}$ \\
\hline $\begin{array}{l}\text { OpenGrey } \\
\text { http://www.opengrey.eu/ }\end{array}$ & (“Cheiloscopy” OR “Lip Print” OR “Lip Pattern” OR “Lipstick”) \\
\hline $\begin{array}{l}\text { OpenThesis } \\
\text { http://www.openthesis.org/ }\end{array}$ & "Cheiloscopy" \\
\hline $\begin{array}{l}\text { Open Access } \\
\text { Theses and Dissertations (OATD) } \\
\text { https://oatd.org/ }\end{array}$ & "Cheiloscopy" \\
\hline
\end{tabular}

Table 1. Strategies for database search.

that most criminals are currently aware of fingerprint analysis and how to avoid leaving such traces in a crime scene-their attention and concern, however, is not the same when it comes to lip prints ${ }^{9}$. Clear-cut furrows that run partially or completely across the lips seem to compose the most prevalent patterns of lip prints, but most of the prevalence studies are restricted to samples that are not even locally representative ${ }^{4}$. Reliable estimates of the presence of lip prints in crime scenes do not exist, but authors progressively endorse this biological trace as "frequent". Soon, studies on cheiloscopy will populate the scientific literature in forensic science and eventually this technique will be presented in Court as means to collect and analyse evidence. It is the role of science to carry out the scrutiny to (I) test the technique, (II) expose to per review, (III) calculate error rates, (IV) promote standardization, and (V) present to the scientific community to verify whether the technique is acceptable-all steps inherent to Daubert's standards.

Considering the existing gap reflected by the uncertainty that surrounds the usefulness of lip print patterns and the urgent need to promote evidence-based science, this study was designed to screen the scientific literature with a systematic approach to find out the real value of cheiloscopy for sexual dimorphism. Prevalence rates of lip print patterns and diagnostic accuracy were the targeted as qualitative and quantitative outcomes of interest.

\section{Materials and methods}

Protocol and registration. This systematic review was performed according to the (1) PRISMA guidelines (Preferred Reporting Items for Systematic Reviews and Meta-Analyses) ${ }^{16}$, (2) the PRISMA standards for Diagnostic Test Accuracy ${ }^{17}$ and (3) the JBI Manual for Evidence Synthesis ${ }^{18}$. The research protocol was submitted for registration at the PROSPERO database.

Focused question. The systematic review followed the acronym PIRD which stands for population (P), index test (I), reference test (R) and diagnosis of interest (D). The guiding research question was: "Is there evidence to determine the biological sex (diagnosis of interest/reference test) of patients free of pathological and/or genetics changes of the lips (population) using cheiloscopy (index test)?”.

Eligibility criteria. Only observational (cohort, case-control and cross-sectional) and diagnostic test accuracy studies were included. No restriction was applied regarding the year or language of publication. The exclusion criteria consisted of studies lacking evident information about the technique used for cheiloscopy, cadaver studies and studies with individuals that had genetic/pathologic alterations of the lip.

Data source and search. The systematic search was performed in August 2020. The primary data sources were Embase, LILACS, PubMed (including MEDLINE), SciELO, Scopus and Web of Science. To avoid/reduce publication bias OpenThesis, OpenGrey and Open Access Theses and Dissertations (OATD) were used as data sources to partially retrieve the grey literature.

Medical Subject Headings (MeSH), Descriptors in Health Sciences (DeCS) and Emtree (Embase Subject Headings) terms were combined by the Boolean operators AND/OR to build search strings (Table 1). Search terms were adapted for each database.

Study selection. Initially, studies were identified after a literature search in each of the databases and imported into EndNote Web (Thomson Reuters, Toronto, Canada) (https://www.myendnoteweb.com) software to remove duplicates. Remaining studies were written down in Microsoft Word 2016 (Microsoft Ltd, Washington, USA) to manually remove duplicates. Next, a training exercise was proposed to reviewers to achieve 
proper agreement during the following phases. The reviewers analyzed $20 \%$ of the studies based on the eligibility criteria. The aimed agreement rate was at least $81 \%$ (Kappa $\geq 0.81$ ). After training, they were able to perform study selection based on title reading (reviewers were not blind for the authorship and year of publication). The next phase consisted of abstract reading and systematic selection. Studies without abstracts available were not excluded in this phase. Finally, the selected studies underwent full-text reading. Studies excluded in this phase had their reason for exclusion registered separately. During all the study selection process, a third reviewer was enrolled to solve any lack of agreement between the two reviewers.

Studies in which the full text could not be retrieved were requested to the authors by e-mail. Additional support was obtained from the Brazilian Program of Bibliographic Commutation (COMUT) and from the Brazilian Institute of Information on Science and Technology (IBICT). In case of studies published in languages other than English, Portuguese and Spanish, the full text was translated.

Data extraction. Data extraction was performed by two examiners independently. A template Microsoft Office Excel (Microsoft Ltd, Washington, USA) sheet was used to assure standardized data extraction. The following data were extracted: (I) identifying information-authorship, year and country of publication of the eligible studies; (II) sample profile-size, age interval, sex distribution and geographic region of origin; (III) cheiloscopy-related data - technique used for analysis, general and sex-related lip print patterns, and sensitivity and specificity of cheiloscopy for sexual dimorphism. Data extraction was supervised by a third reviewer and a forensic odontologist.

The corresponding authors were contacted by email (up to three times over two weeks) to obtain relevant information in case of missing or unclear data.

Risk of bias. The risk of bias and the assessment of individual methodological quality of the eligible studies were accomplished by means of JBI Critical Appraisal tool for observational cross-sectional ${ }^{19}$ or diagnostic test accuracy $^{20}$ studies. Following PRISMA ${ }^{16}$, two reviewers assessed the risk of bias. Lack of agreement between reviewers for any of the questions within the JBI tool was solved by a third examiner.

The percentage of positive answers to the questions led to the final score of the studies. Studies that scored up to $49 \%$ of positive answers were classified as "high risk of bias". Studies with positive answers between 50 and $69 \%$ were classified as "moderate risk of bias", while studies that scored positive answers above $70 \%$ were classified as "low risk of bias".

\section{Summary measures}

The outcomes were explored by means of descriptive analysis and were presented in narrative tables. The prevalence of lip print patterns was reported according to sex and compared between males and females. More specifically, this analysis was performed using a meta-analytical approach of proportions, in which combined prevalence estimates for males and females were estimated using random effects and Freeman-Tukey double transformation to stabilize the model's variances ${ }^{21}$. The heterogeneity between groups was estimated to assess the differences of lip print patterns between males and females. A meta-analysis was adjusted for each combination of lip print pattern, lip side (right/left) and lip position (upper lower). Studies with missing information about lip print pattern, lip side and lip position were not included in the meta-analysis. The meta-analysis was performed separately for the two predominant techniques found in the systematic literature review: Suzuki \& Tsuchihasi (1970) and Renaud (1973).

The diagnostic accuracy of the cheiloscopy technique for sexual dimorphism was tested separately for males and females. The absolute number of correct match and mismatch between reference and target lips was extracted from each eligible study and a meta-analysis using random effect was adjusted. To avoid the exclusion of studies that reported zero match or mismatch, a correction of continuity of 0.5 was established in these cases. Studies that provided the number of hits and errors for males and females separately were included in a meta-analysis evaluating if the accuracy of cheiloscopy differed in distinguishing males and females. To assess that, the odds ratio for identifying males compared to females was calculated, and it evaluated if the methods was more or less accurate for sexual dimorphism among males compared to females.

For meta-analyses that included at least 10 studies, publication bias was investigated through Egger's test by a linear regression of the effect measure on the size of the study ${ }^{22}$. Statistical analyses were performed with Stata version 16.1 (StataCorp LLC, College Station, TX, USA) software. Significance level was set at 5\%.

Certainty of evidence (GRADE approach). Certainty of evidence and strength of recommendation were assessed with the Grading of Recommendation, Assessment, Development, and Evaluation (GRADE) approach. According to this system, diagnostic accuracy studies start at a high level of certainty and can be downgraded based on risk of bias, inconsistency, indirect evidence, imprecision, and publication bias. The level of certainty among the identified evidence was characterized as high, moderate, low, or very low ${ }^{23}$.

\section{Results}

Study selection. The first phase of study selection resulted in 3,977 studies throughout the nine electronic databases. After removing duplicates, the remaining number of studies was 2,956. Exclusions based on title and abstract reading reduced the sample to 98 studies eligible for full-text reading. Six studies did not fulfill the inclusion criteria (Appendix 1), and full texts were not found for twenty studies, even after trying to contact the authors or libraries. Finally, a total of 72 studies were selected for qualitative analysis ${ }^{1,2,4-6,10-15,24-84}$. Quantitative analysis of the accuracy of cheiloscopy for sexual dimorphism included seven studies ${ }^{1,5,25,48,49,54,80}$, and 17 


\section{Identification of studies via databases and registers}
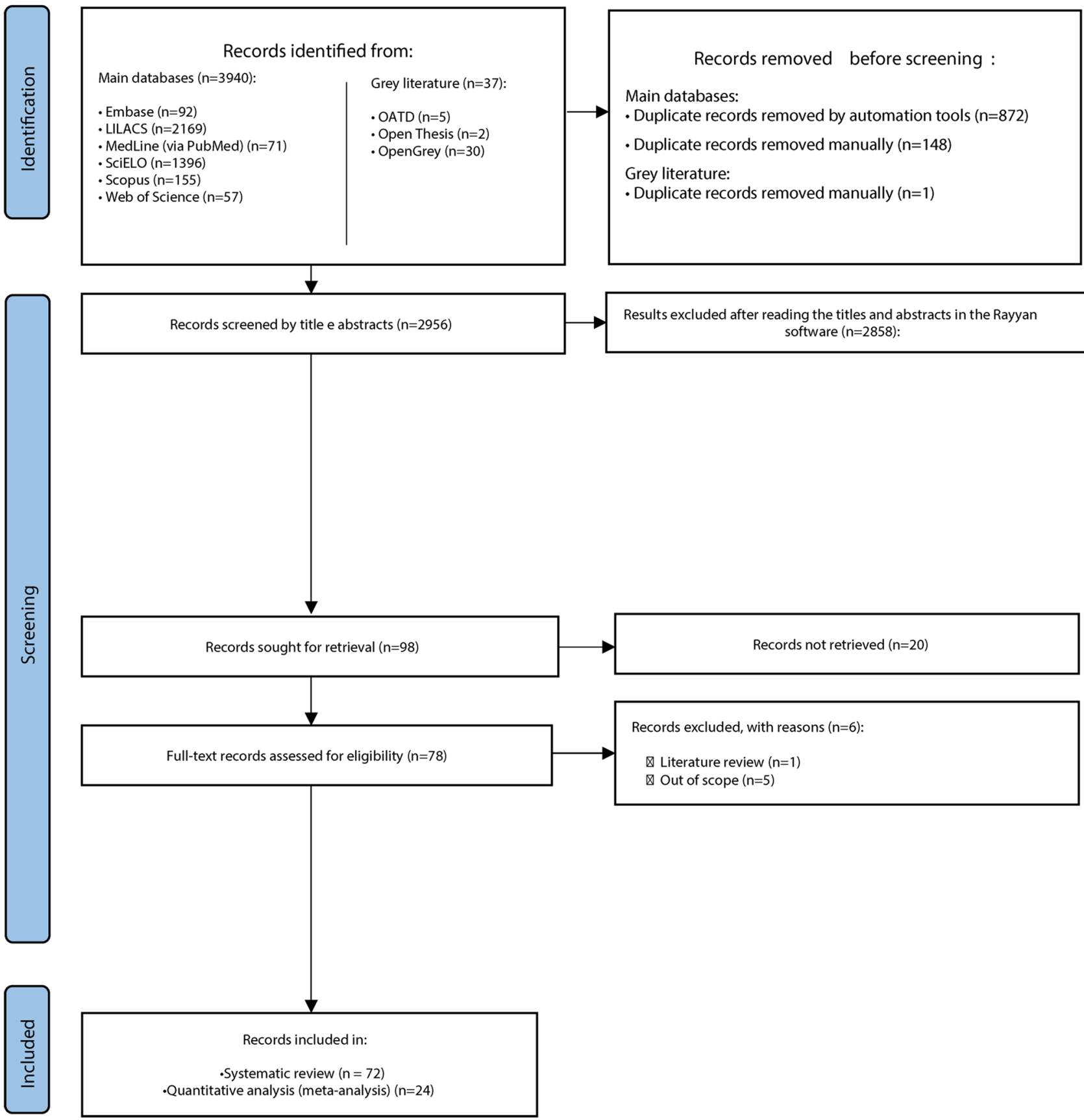

Figure 1. Flowchart diagram, following PRISMA, describing the quantity of studies filtered from identification to the final inclusion in the qualitative and quantitative (meta-) analyses.

studies $^{10,11,14,28,30,32,34,36,38,42,43,51,56,60,61,63,82}$ were considered in the analyses of the prevalence of lip print patterns (Fig. 1).

Characteristics of eligible studies. The studies were published between 1982 and 2019, and were from India $(\mathrm{n}=52)^{1,4-6,10-15,25,27,30,31,35-40,43-46,48-50,53-57,59-62,64,66-75,78,79,81,83,84}$, Egypt $(\mathrm{n}=3)^{2,42,58}$, Brazil $(\mathrm{n}=3)^{26,34,76}$, Portugal $(n=2)^{32,51}$, Pakistan $(n=2)^{47,77}$, Colombia $(n=2)^{29,52}$, Nepal $(n=2)^{33,82}$, France $(n=1)^{24}$, Iran $(n=1)^{63}$, Romania $(n=1)^{41}$, Croatia $(n=1)^{65}$, Saudi Arabia $(n=1)^{28}$ and Poland $(n=1)^{80}$. The total sample of participants across studies was 22,965. The age interval of the of participants ranged from 1 to 83 years (Table 2). Fourteen studies did not describe the ethical aspects adopted in the study. None of the cross-sectional studies reported STROBE checklist as the guideline of choice. 


\begin{tabular}{|c|c|c|c|c|c|}
\hline Authors, year ${ }^{\text {ref }}$ & Country & Age (years) & n & Technique & Data collection \\
\hline Fauvel et al., $1982^{24}$ & France & $3-73$ & $111\left(42 \delta^{\wedge} ; 69+\right)$ & Fauvel's & Polymer and varnish \\
\hline Sonal et al., $2005^{25}$ & India & $19-29$ & $50\left(20{ }^{\hat{\gamma}} ; 30\right.$ 央 $)$ & Suzuki and Tsuchihashi's & Lipstick and paper \\
\hline Barros, $2006^{26}$ & Brazil & $\mathrm{n} / \mathrm{r}$ & $120(60 \bigcirc ; 60+)$ & Suzuki and Tsuchihashi's & Lipstick, paper and photographs \\
\hline Augustine et al., $2008^{10}$ & India & $3-83$ & $600(280 \bigcirc ; 320+)$ & Suzuki and Tsuchihashi's & Lipstick and paper digitized \\
\hline Sharma and Saxena, $2009^{27}$ & India & $\mathrm{n} / \mathrm{r}$ & $100\left(50{ }^{\hat{\gamma}} ; 50+\right.$ + $)$ & Suzuki and Tsuchihashi's & Lipstick and paper \\
\hline El Domiaty et al., $2010^{28}$ & Saudi Arabia & $18-40$ & $966(426 \widehat{3} ; 540+$ + $)$ & Renaud's & Lipstick, paper and photographs \\
\hline Chalapud et al., $2011^{29}$ & Colombia & $17-30$ & $47\left(23{ }^{3} ; 24+9\right)$ & Renaud's & Lipstick, paper and photographs \\
\hline Gupta et al., $2011^{30}$ & India & $18-30$ & $146(73 \precsim ; 33$ 우 $)$ & Suzuki and Tsuchihashi's & Lipstick and paper \\
\hline Prasad and Vanishree, $2011^{31}$ & India & $17-21$ & $100(50 \bigcirc ; 50+$ 웃 & Suzuki and Tsuchihashi's & Lipstick and paper \\
\hline Amith et al., $2012^{11}$ & India & $10-25$ & $1539\left(695 \jmath^{\lambda} ; 844\right.$ 우 $)$ & Suzuki and Tsuchihashi's & Lipstick and paper \\
\hline Babladi et al., $2012^{12}$ & India & $18-22$ & $124(66 \overbrace{}^{\lambda} ; 58$ 우 $)$ & Suzuki and Tsuchihashi's & Lipstick and paper \\
\hline Costa and Caldas, $2012^{32}$ & Portugal & $20-33$ & $50\left(25{ }^{\wedge} ; 25 ㅇ ㅜ\right)$ & Suzuki and Tsuchihashi's & Lipstick and paper digitized \\
\hline Karki, $2012^{33}$ & Nepal & $18-25$ & $150(75 \delta ; 75 ㅇ)$ & Suzuki and Tsuchihashi's & Lipstick and paper \\
\hline Oliveira et al., $2012^{34}$ & Brazil & $\mathrm{n} / \mathrm{r}$ & $104(54 \bigcirc ; 50$ ? $)$ & Suzuki and Tsuchihashi's & Lipstick, paper and photographs \\
\hline Prabhu et al., $2012^{35}$ & India & $19-28$ & $100(ð+\mathrm{n} / \mathrm{r})$ & Suzuki and Tsuchihashi's & Lipstick, paper and scanning \\
\hline Rastogi and Parida, $2012^{36}$ & India & $18-25$ & $100\left(\jmath^{\wedge}+\mathrm{n} / \mathrm{r}\right)$ & Suzuki and Tsuchihashi's & Lipstick and paper \\
\hline Vats et al., $2012^{37}$ & India & $8-60$ & $1399(781 \delta ; 618$ 우 & Suzuki and Tsuchihashi's & Lipstick and paper \\
\hline Bansal et al., $2013^{6}$ & India & $20-50$ & $5000(2500 \overbrace{}^{\wedge} ; 2500$ ㅇ $)$ & Suzuki and Tsuchihashi's & Lipstick and paper \\
\hline Kautilya et al., $2013^{38}$ & India & $18-25$ & $100(50 \overbrace{}^{\wedge} ; 50$ 古 $)$ & Suzuki and Tsuchihashi's & Lipstick and paper \\
\hline Koneru et al., $2013^{39}$ & India & $18-21$ & $60\left(30{ }^{\Uparrow} ; 30\right.$ 우 $)$ & Suzuki and Tsuchihashi's & Lipstick and paper \\
\hline Padmavathi et al., $2013^{40}$ & India & $\mathrm{n} / \mathrm{r}$ & $250($ & Suzuki and Tsuchihashi's & Lipstick, paper and photographs \\
\hline Popa et al., $2013^{41}$ & Romania & $24-37$ & $100\left(50{ }^{\wedge} ; 50\right.$ ㅇ $)$ & Suzuki and Tsuchihashi's & Lipstick and paper \\
\hline Ragab et al., $2013^{42}$ & Egypt & $2-65$ & $955\left(235 \delta^{\lambda} ; 720 ㅇ\right)$ & Renaud's & Lipstick, paper and scanning \\
\hline Sekhon et al., $2013^{43}$ & India & $\mathrm{n} / \mathrm{r}$ & $300\left(100{ }^{\Uparrow} ; 200\right.$ 우 $)$ & Suzuki and Tsuchihashi's & Lipstick, paper and scanning \\
\hline Verma et al., $2013^{44}$ & India & $18-25$ & $208(85 \delta ; 123$ 우 $)$ & Suzuki and Tsuchihashi's & Lipstick and paper \\
\hline Gupta et al., $2014^{45}$ & India & $18-30$ & $378(189 \bigcirc ; 189+9)$ & Suzuki and Tsuchihashi's & Lipstick and paper \\
\hline Hammad et al., $2014^{46}$ & Pakistan & $19-25$ & $100(30 \overbrace{}^{\lambda} ; 70$ + $)$ & Suzuki and Tsuchihashi's & Lipstick and paper \\
\hline Multani et al., $2014^{47}$ & India & $15-55$ & $200\left(100{ }^{\Uparrow} ; 100+9\right)$ & Suzuki and Tsuchihashi's & Lipstick and paper \\
\hline Nagalaxmi et al., $2014^{48}$ & India & $20-30$ & $60(30 \overbrace{}^{\wedge} ; 30$ + $)$ & Suzuki and Tsuchihashi's & Lipstick and paper \\
\hline Ramaligam et al., $2014^{49}$ & India & $20-30$ & $40(20 \overbrace{}^{\lambda} ; 20$ 早 $)$ & Suzuki and Tsuchihashi's & Lipstick and paper \\
\hline Sharma et al., $2014^{5}$ & India & $17-26$ & $200\left(100{ }^{\wedge} ; 100+9\right)$ & Suzuki and Tsuchihashi's & Lipstick and paper \\
\hline Abidullah et al., $2015^{13}$ & India & $18-30$ & $200(100 \overbrace{}^{\wedge} ; 100 ;$; + ) & Suzuki and Tsuchihashi's & Lipstick and paper \\
\hline Bharathi and Thenmozhi, $2015^{51}$ & India & $\mathrm{n} / \mathrm{r}$ & $100(24 \bigcirc ; 76$ 角 $)$ & Suzuki and Tsuchihashi's & Lipstick and paper \\
\hline Cartaxo, $2015^{52}$ & Portugal & $17-40$ & $202(94 \bigcirc ; 108$ ? $)$ & Suzuki and Tsuchihashi's & Lipstick, paper and photographs \\
\hline Hernández et al., $2015^{53}$ & Colombia & $18-25$ & $60(30 \overbrace{}^{\wedge} ; 30+)$ & Suzuki and Tsuchihashi's & Lipstick and paper \\
\hline Kaul et al., $2015^{54}$ & India & $1-80$ & $755\left(375 \jmath^{\wedge} ; 380\right.$ 우) & Suzuki and Tsuchihashi's & Lipstick and paper \\
\hline Nagpal et al., $2015^{55}$ & India & $18-24$ & $60(20 \overbrace{}^{\Uparrow} ; 40$ 우 $)$ & Suzuki and Tsuchihashi's & Lipstick and paper \\
\hline Peeran et al., $2015^{56}$ & India & $18-35$ & $104\left(37 \jmath^{\prime} ; 67 ㅇ ㅜ\right)$ & Suzuki and Tsuchihashi's & Lipstick and paper \\
\hline Shah and Jayaraj, $2015^{57}$ & India & $17-25$ & $200(100 \bigcirc ; 100$ 우 $)$ & Suzuki and Tsuchihashi's & Lipstick and paper \\
\hline Sharma et al., $2015^{58}$ & India & $18-25$ & $201\left(107 \delta \gamma^{\prime} ; 94\right.$ + $)$ & Suzuki and Tsuchihashi's & Lipstick and paper \\
\hline Badiye and Kapoor, $2016^{50}$ & India & $18-25$ & $400(200 \overbrace{}^{\wedge} ; 200+$ + $)$ & Suzuki and Tsuchihashi's & Lipstick and photographs \\
\hline Aziz et al., $2016^{59}$ & Egypt & $\mathrm{n} / \mathrm{r}$ & $120\left(60 \delta^{\wedge} ; 60+\right)$ & Suzuki and Tsuchihashi's & Lipstick and paper \\
\hline Borase et al., $2016^{60}$ & India & $20-50$ & $496\left(326{ }^{\wedge} ; 170+9\right)$ & Renaud's & Lipstick and paper digitized \\
\hline Jeergal et al., $2016^{61}$ & India & $18-60$ & $200(100 \bigcirc ; 100$ 우 & Suzuki and Tsuchihashi's & Lipstick and paper digitized \\
\hline Krishnan et al., $2016^{62}$ & India & $18-21$ & $60(30 今 ; 30$ 우 $)$ & Suzuki and Tsuchihashi's & Lipstick and paper \\
\hline Moshfeghi et al., $2016^{63}$ & Iran & $13-70$ & $96(22 \overbrace{}^{\wedge} ; 74$ 우 $)$ & Suzuki and Tsuchihashi's & Lipstick and paper \\
\hline Negi and Negi, $2016^{64}$ & India & $\mathrm{n} / \mathrm{r}$ & $200\left(100{ }^{\Uparrow} ; 100+\right.$ + $)$ & Nagasupriya’s & Lipstick and paper \\
\hline Simovic et al., $2016^{65}$ & Croatia & $\mathrm{n} / \mathrm{r}$ & $90\left(40{ }^{\wedge} ; 50\right.$ 우 $)$ & Suzuki and Tsuchihashi's & Lipstick and paper \\
\hline Tarvadi and Goyal, $2016^{66}$ & India & $18-25$ & $100\left(50{ }^{\wedge} ; 50+9\right)$ & Suzuki and Tsuchihashi's & Lipstick and paper \\
\hline Alzapur et al., $2017^{4}$ & India & $17-19$ & $100(50 \overbrace{}^{\wedge} ; 50$ 운 & Suzuki and Tsuchihashi's & Lipstick and paper \\
\hline Basheer et al., $2017^{14}$ & India & $18-30$ & $858\left(471 \jmath^{\Uparrow} ; 387 ㅇ ㅜ\right)$ & Suzuki and Tsuchihashi's & Lipstick and paper \\
\hline Kumar, $2017^{67}$ & India & $10-16$ & $200\left(100{ }^{\wedge} ; 100\right.$ 우 $)$ & Suzuki and Tsuchihashi's & Lipstick and paper \\
\hline Chaudhari et al., $2017^{68}$ & India & $25-50$ & $150\left(75 \jmath^{2} 75+9\right)$ & Suzuki and Tsuchihashi's & Lipstick and paper \\
\hline Gouda and Rao, $2017^{69}$ & India & $18-23$ & $100\left(50{ }^{\wedge} 50\right.$ 우 $)$ & Suzuki and Tsuchihashi's & Lipstick and paper \\
\hline Kapoor and Badyie, $2017^{70}$ & India & $18-25$ & $200(100 \overbrace{}^{\lambda} 100$ 우 $)$ & Suzuki and Tsuchihashi's & Lipstick and photographs \\
\hline Naik et al., $2017^{71}$ & India & $18-20$ & $100\left(50{ }^{\wedge} ; 50\right.$ 우 $)$ & Suzuki and Tsuchihashi's & Lipstick and Whatman paper filter \\
\hline
\end{tabular}




\begin{tabular}{|c|c|c|c|c|c|}
\hline Authors, year ${ }^{\text {ref }}$ & Country & Age (years) & $\mathbf{n}$ & Technique & Data collection \\
\hline Sandhu et al., $2017^{72}$ & India & $18-30$ & $1200\left(540{ }^{\wedge} ; 660\right.$ ㅇ $)$ & Suzuki and Tsuchihashi's & Lipstick and paper \\
\hline Tandon et al., $2017^{73}$ & India & $20-50$ & $100(50 \overbrace{}^{\wedge} ; 50$ 古 $)$ & Suzuki and Tsuchihashi's & Lipstick and paper \\
\hline Vignesh et al., $2017^{74}$ & India & $3-6$ & $300(\widehat{\jmath}+\mathrm{n} / \mathrm{r})$ & Suzuki and Tsuchihashi's & Lipstick and paper \\
\hline Ahmed et al., $2018^{2}$ & Egypt & $26.8 \pm 10.4$ & $221(105 \widehat{\beta} ; 116+)$ & Suzuki and Tsuchihashi's & Lipstick and paper \\
\hline Bai et al., $2018^{15}$ & India & $18-25$ & $300\left(150{ }^{\circledR} ; 150\right.$ 우) & Suzuki and Tsuchihashi's & Lipstick and paper \\
\hline Herrera et al., $2018^{75}$ & Brazil & $18-71$ & $50\left(25 \jmath^{\wedge} ; 25+\right.$ ㅇ $)$ & Suzuki and Tsuchihashi's & Lipstick, CD, glass and photographs \\
\hline Ishaq et al., $2018^{76}$ & Pakistan & $\mathrm{n} / \mathrm{r}$ & $250\left(125{ }^{\prime} ; 125 ㅇ\right)$ & Suzuki and Tsuchihashi's & Lipstick and paper \\
\hline Manikya et al., $2018^{77}$ & India & $18-23$ & $180\left(90{ }^{\prime} ; 90\right.$ 우) & Suzuki and Tsuchihashi's & Lipstick and paper \\
\hline Bhagyashree et al., $2018^{78}$ & India & $18-30$ & $100\left(50{ }^{3} ; 50\right.$ 우 $)$ & Suzuki and Tsuchihashi's & Lipstick, paper and glass \\
\hline Thomas et al., $2018^{79}$ & India & $18-26$ & $128(67 \hat{\jmath} ; 61$ \&) & Suzuki and Tsuchihashi's & Lipstick and paper \\
\hline Topczyłko et al., $2018^{80}$ & Poland & $15-30$ & $242(76 \hat{\jmath} ; 166+9)$ & Suzuki and Tsuchihashi's, Renaud's, Vahanwala's & $\mathrm{n} / \mathrm{r}$ \\
\hline Bansal et al., $2019^{1}$ & India & $18-21$ & $200\left(100{ }^{\gamma} ; 100 ㅇ\right)$ & Suzuki and Tsuchihashi's & Lipstick, paper, glass and powder \\
\hline Divyadharsini and Kumar, $2019^{81}$ & India & $20-30$ & $100\left(50{ }^{\prime} ; 50\right.$ 우 $)$ & Suzuki and Tsuchihashi's & Lipstick and paper \\
\hline Gurung et al., $2019^{82}$ & Nepal & $17-24$ & $205\left(141 \delta_{\delta}^{\wedge} 64\right.$ 우 $)$ & Suzuki and Tsuchihashi's & Lipstick and paper \\
\hline Vaishnavi et al., $2019^{83}$ & India & $15-20$ & $50\left(25 \delta^{\wedge} ; 25\right.$ 우 $)$ & $\mathrm{n} / \mathrm{r}$ & Lipstick and paper \\
\hline Yendriwati et al., $2019^{84}$ & India & $20-26$ & $30\left(15 \delta^{\wedge} ; 15\right.$ 우 $)$ & Suzuki and Tsuchihashi's & Lipstick and paper \\
\hline
\end{tabular}

Table 2. Main characteristics of eligible studies. $\sigma^{\lambda}$ : Male ${ }_{+}$: Female; n/r: Not reported by the authors.

\begin{tabular}{|c|c|c|c|c|c|c|c|c|c|c|c|c|}
\hline Study & Q.1 & Q.2 & Q.3 & Q.4 & Q.5 & Q.6 & Q.7 & Q.8 & Q.9 & Q.10 & \% Yes & Risk \\
\hline Sonal et al., $2005^{25}$ & - & - & $\checkmark$ & $\checkmark$ & N/A & $\checkmark$ & N/A & N/A & $\checkmark$ & $\checkmark$ & & Low \\
\hline Nagalaxmi et al., $2014^{48}$ & - & - & $\checkmark$ & $\mathrm{U}$ & N/A & $\checkmark$ & N/A & N/A & $\checkmark$ & $\checkmark$ & & Moderate \\
\hline Ramaligam et al., $2014^{49}$ & - & - & $\checkmark$ & $\checkmark$ & N/A & $\checkmark$ & N/A & N/A & $\checkmark$ & $\checkmark$ & & Low \\
\hline Sharma et al., $2014^{5}$ & $\mathrm{U}$ & - & $\checkmark$ & $\checkmark$ & N/A & $\checkmark$ & N/A & N/A & $\checkmark$ & $\checkmark$ & & Low \\
\hline Kaul et al., $2015^{54}$ & $\mathrm{U}$ & - & $\checkmark$ & $\checkmark$ & N/A & $\checkmark$ & N/A & N/A & $\checkmark$ & $\checkmark$ & & Low \\
\hline Topczyłko et al., $2018^{80}$ & $\mathrm{U}$ & - & $\checkmark$ & $\mathrm{U}$ & N/A & $\checkmark$ & N/A & $\mathrm{N} / \mathrm{A}$ & $\checkmark$ & $\checkmark$ & & Moderate \\
\hline Bansal et al., $2019^{1}$ & $\mathrm{U}$ & - & $\checkmark$ & $\mathrm{U}$ & N/A & $\checkmark$ & N/A & N/A & $\checkmark$ & $\checkmark$ & & Moderate \\
\hline
\end{tabular}

Table 3. Risk of bias assessed by the Joanna Briggs Institute Critical Appraisal Tools for use in JBI Critical Appraisal Checklist for Diagnostic Accuracy Studies. Q.1. Was a consecutive or random sample of patients enrolled? Q.2. Was a case control design avoided? Q. 3. Did the study avoid inappropriate exclusions? Q.4. Were the index test results interpreted without knowledge of the results of the reference standard? Q.5. If a threshold was used, was it pre-specified? Q.6. Is the reference standard likely to correctly classify the target condition? Q.7. Were the reference standard results interpreted without knowledge of the results of the index test? Q.8. Was there an appropriate interval between index test and reference standard? Q.9. Did all patients receive the same reference standard? Q.10. Were all patients included in the analysis? $\checkmark$ : Yes; -: No; U : Unclear: N/A: Not applicable.

Sixty-four studies ${ }^{1,2,4-6,10-15,25-27,30-41,43-59,61-63,65-79,81,82,84}$ used the technique of Suzuki and Tsuchihashi (1970), four studies ${ }^{28,29,42,60}$ used Renaud's (1973) technique, one study ${ }^{24}$ used Fauvel's (1985) technique, one study ${ }^{64}$ used Nagasupriya's (2011) technique, and one study ${ }^{80}$ combined the techniques of Suzuki and Tsuchihashi (1970), Renaud (1973), and Vahanwala (2000). One study ${ }^{83}$ did not report which technique was used. In general, twentyfour studies $(33 \%)^{12,14,24,26,28,31,35,42-44,47,51,53-55,57,63,66,67,74-76,82}$ did not find evidence of difference of lip print patterns between males and females, while $67 \%^{1,2,4-6,10,11,13,15,25,27,29,31-34,36-41,45,46,48-50,52,56,58-62,64,65,68-73,77-81,83,84}$ detected differences.

Individual risk of bias. Fifty eligible studies $2,4,5,10,14,25,26,28-30,32,35,38-40,42,44-46,49-58,60-63,66-72,74,76-79,81-84$ had low risk of bias, while 22 studies $^{1,6,11-13,15,24,27,31,33,36,37,41,43,47,48,59,64,65,73,75,80}$ had moderate risk of bias (Tables 3 and 4). All the questions in JBI tool for cross-sectional studies were applicable, while three questions were not applicable in the JBI tool for diagnostic test accuracy studies.

Regarding cross-sectional studies, questions \#5 and \#6 had a negative answer in 25 studies ${ }^{6,11-13,15,24,26,27,30,31,33,35,36,39,41,43,47,50,55,57,64,66-68,71,73,83}$. These questions verify if the study identified and avoided confounding factors, since studies should minimize the risk of bias describing factors that could influence on the process of collecting lip print evidence. In 28 studies $^{2,6,10,11,13,15,24,26,27,30,31,33,35,36,39,41,43,47,50,55,57,64,66-68,71,73,83}$ question \#7 had a negative answer. This question has a direct impact in the quality of the evidence because it verifies if the outcomes were obtained in a reliable way. An example of attitude towards a positive answer is the minimization of bias by describing the process of intra- and inter-examiner training.

Concerning diagnostic test accuracy studies, questions \#1 and \#2 were marked as 'unclear' or 'no' for all studies $1,5,25,48,49,54,80$. The first question checked whether the sample was selected consecutively or randomly. The second question was related to the methodological design of the studies; all studies recruited participants that 


\begin{tabular}{|c|c|c|c|c|c|c|c|c|c|c|}
\hline Authors & Q1 & Q2 & Q3 & Q4 & Q5 & Q6 & Q7 & Q8 & \% Yes & Risk \\
\hline Fauvel et al., $1982^{24}$ & $\checkmark$ & $\checkmark$ & $\checkmark$ & $\checkmark$ & - & - & - & $\checkmark$ & 62.5 & Moderate \\
\hline Barros, $2006^{26}$ & $\checkmark$ & $\checkmark$ & $\checkmark$ & $\checkmark$ & $\checkmark$ & $\checkmark$ & - & $\checkmark$ & 87.5 & Low \\
\hline Augustine et al., $2008^{10}$ & - & $\checkmark$ & $\checkmark$ & $\checkmark$ & - & - & $\checkmark$ & $\checkmark$ & 62.5 & Moderate \\
\hline Sharma and Saxena, $2009^{27}$ & $\checkmark$ & $\checkmark$ & $\checkmark$ & $\checkmark$ & - & - & - & $\checkmark$ & 62.5 & Moderate \\
\hline El Domiaty et al., $2010^{28}$ & $\checkmark$ & $\checkmark$ & $\checkmark$ & $\checkmark$ & - & - & $\checkmark$ & $\checkmark$ & 75 & Low \\
\hline Chalapud et al., $2011^{29}$ & - & $\checkmark$ & $\checkmark$ & $\checkmark$ & $\checkmark$ & - & $\checkmark$ & $\checkmark$ & 75 & Low \\
\hline Gupta et al., $2011^{30}$ & $\checkmark$ & $\checkmark$ & $\checkmark$ & $\checkmark$ & $\checkmark$ & $\checkmark$ & - & $\checkmark$ & 87.5 & Low \\
\hline Prasad and Vanishree, $2011^{31}$ & - & $\checkmark$ & $\checkmark$ & $\checkmark$ & - & - & - & $\checkmark$ & 50 & Moderate \\
\hline Amith et al., $2012^{11}$ & $\checkmark$ & $\checkmark$ & $\checkmark$ & $\checkmark$ & - & - & - & $\checkmark$ & 62.5 & Moderate \\
\hline Babladi et al., $2012^{12}$ & $\checkmark$ & $\checkmark$ & $\checkmark$ & $\checkmark$ & - & - & - & $\checkmark$ & 62.5 & Moderate \\
\hline Costa and Caldas, $2012^{32}$ & $\checkmark$ & $\checkmark$ & $\checkmark$ & $\checkmark$ & $\checkmark$ & $\checkmark$ & $\checkmark$ & $\checkmark$ & 100 & \begin{tabular}{|l|} 
Low \\
\end{tabular} \\
\hline Karki, $2012^{33}$ & - & $\checkmark$ & $\checkmark$ & $\checkmark$ & - & - & - & $\checkmark$ & 50 & Moderate \\
\hline Oliveira et al., $2012^{34}$ & $\checkmark$ & - & $\checkmark$ & $\checkmark$ & $\checkmark$ & $\checkmark$ & $\checkmark$ & $\checkmark$ & 87.5 & Low \\
\hline Prabhu et al., $2012^{35}$ & $\checkmark$ & $\checkmark$ & $\checkmark$ & $\checkmark$ & $\checkmark$ & $\checkmark$ & - & $\checkmark$ & 87.5 & Low \\
\hline Rastogi and Parida, $2012^{36}$ & $\checkmark$ & $\checkmark$ & $\checkmark$ & $\checkmark$ & - & - & - & $\checkmark$ & 62.5 & Moderate \\
\hline Vats et al., 2012 ${ }^{37}$ & - & - & $\checkmark$ & $\checkmark$ & - & - & $\checkmark$ & $\checkmark$ & 50 & Moderate \\
\hline Bansal et al., $2013^{6}$ & - & - & $\checkmark$ & $\checkmark$ & - & $\checkmark$ & - & $\checkmark$ & 50 & Moderate \\
\hline Kautilya et al., $2013^{38}$ & $\checkmark$ & $\checkmark$ & $\checkmark$ & $\checkmark$ & - & - & $\checkmark$ & $\checkmark$ & 75 & \begin{tabular}{|l|} 
Low \\
\end{tabular} \\
\hline Koneru et al., $2013^{39}$ & $\checkmark$ & $\checkmark$ & $\checkmark$ & $\checkmark$ & $\checkmark$ & $\checkmark$ & - & $\checkmark$ & 87.5 & Low \\
\hline Padmavathi et al., $2013^{40}$ & - & $\checkmark$ & - & $\checkmark$ & - & - & $\checkmark$ & $\checkmark$ & 75 & Low \\
\hline Popa et al., $2013^{41}$ & $\checkmark$ & $\checkmark$ & $\checkmark$ & $\checkmark$ & - & - & - & $\checkmark$ & 62.5 & Moderate \\
\hline Ragab et al., $2013^{42}$ & $\checkmark$ & $\checkmark$ & $\checkmark$ & $\checkmark$ & $\checkmark$ & $\checkmark$ & $\checkmark$ & $\checkmark$ & 100 & Low \\
\hline Sekhon et al., $2013^{43}$ & $\checkmark$ & $\checkmark$ & $\checkmark$ & $\checkmark$ & - & - & - & $\checkmark$ & 62.5 & Moderate \\
\hline Verma et al., $2013^{44}$ & $\checkmark$ & $\checkmark$ & $\checkmark$ & $\checkmark$ & - & - & $\checkmark$ & $\checkmark$ & 75 & Low \\
\hline Gupta et al., $2014^{45}$ & $\checkmark$ & $\checkmark$ & $\checkmark$ & $\checkmark$ & $\checkmark$ & $\checkmark$ & $\checkmark$ & $\checkmark$ & 100 & Low \\
\hline Hammad et al., $2014^{46}$ & $\checkmark$ & $\checkmark$ & $\checkmark$ & $\checkmark$ & - & - & - & $\checkmark$ & 62.5 & Moderate \\
\hline Multani et al., $2014^{47}$ & $\checkmark$ & $\checkmark$ & $\checkmark$ & $\checkmark$ & $\checkmark$ & $\checkmark$ & $\checkmark$ & $\checkmark$ & 100 & Low \\
\hline Abidullah et al., $2015^{13}$ & $\checkmark$ & $\checkmark$ & $\checkmark$ & $\checkmark$ & $\checkmark$ & $\checkmark$ & - & $\checkmark$ & 87.5 & Low \\
\hline Bharathi and Thenmozhi, $2015^{51}$ & $\checkmark$ & - & $\checkmark$ & $\checkmark$ & $\checkmark$ & $\checkmark$ & - & $\checkmark$ & 75 & Low \\
\hline Cartaxo, $2015^{52}$ & $\checkmark$ & $\checkmark$ & $\checkmark$ & $\checkmark$ & $\checkmark$ & $\checkmark$ & $\checkmark$ & $\checkmark$ & 100 & Low \\
\hline Hernández et al., $2015^{53}$ & $\checkmark$ & $\checkmark$ & $\checkmark$ & $\checkmark$ & - & - & $\checkmark$ & $\checkmark$ & 75 & Low \\
\hline Nagpal et al., $2015^{55}$ & $\checkmark$ & $\checkmark$ & $\checkmark$ & $\checkmark$ & $\checkmark$ & $\checkmark$ & $\checkmark$ & $\checkmark$ & 100 & Low \\
\hline Peeran et al., $2015^{56}$ & $\checkmark$ & $\checkmark$ & $\checkmark$ & $\checkmark$ & $\checkmark$ & $\checkmark$ & - & $\checkmark$ & 87.5 & Low \\
\hline Shah and Jayaraj, $2015^{57}$ & $\checkmark$ & $\checkmark$ & $\checkmark$ & $\checkmark$ & $\checkmark$ & $\checkmark$ & $\checkmark$ & $\checkmark$ & 100 & Low \\
\hline Sharma et al., $2015^{58}$ & $\checkmark$ & $\checkmark$ & $\checkmark$ & $\checkmark$ & $\checkmark$ & $\checkmark$ & - & $\checkmark$ & 87.5 & Low \\
\hline Badiye and Kapoor, $2016^{50}$ & - & $\checkmark$ & $\checkmark$ & $\checkmark$ & - & - & $\checkmark$ & $\checkmark$ & 62.5 & Moderate \\
\hline Aziz et al., $2016^{59}$ & $\checkmark$ & $\checkmark$ & $\checkmark$ & $\checkmark$ & $\checkmark$ & $\checkmark$ & $\checkmark$ & $\checkmark$ & 100 & Low \\
\hline Borase et al., $2016^{60}$ & $\checkmark$ & $\checkmark$ & $\checkmark$ & $\checkmark$ & $\checkmark$ & $\checkmark$ & $\checkmark$ & $\checkmark$ & 100 & Low \\
\hline Jeergal et al., $2016^{61}$ & $\checkmark$ & - & $\checkmark$ & $\checkmark$ & $\checkmark$ & $\checkmark$ & $\checkmark$ & $\checkmark$ & 87.5 & Low \\
\hline Krishnan et al., $2016^{62}$ & $\checkmark$ & $\checkmark$ & $\checkmark$ & $\checkmark$ & $\checkmark$ & $\checkmark$ & $\checkmark$ & $\checkmark$ & 100 & Low \\
\hline Moshfeghi et al., $2016^{63}$ & $\checkmark$ & $\checkmark$ & $\checkmark$ & $\checkmark$ & $\checkmark$ & $\checkmark$ & $\checkmark$ & $\checkmark$ & 100 & Low \\
\hline Negi and Negi, $2016^{64}$ & - & - & $\checkmark$ & $\checkmark$ & $\checkmark$ & $\checkmark$ & - & $\checkmark$ & 62.5 & Moderate \\
\hline Simovic et al., $2016^{65}$ & - & - & $\checkmark$ & $\checkmark$ & - & - & $\checkmark$ & $\checkmark$ & 50 & Moderate \\
\hline Tarvadi and Goyal, $2016^{66}$ & $\checkmark$ & $\checkmark$ & $\checkmark$ & $\checkmark$ & $\checkmark$ & $\checkmark$ & - & $\checkmark$ & 87.5 & Low \\
\hline Alzapur et al., $2017^{4}$ & $\checkmark$ & $\checkmark$ & $\checkmark$ & $\checkmark$ & $\checkmark$ & $\checkmark$ & $\checkmark$ & $\checkmark$ & 100 & \begin{tabular}{|l|} 
Low \\
\end{tabular} \\
\hline Basheer et al., $2017^{14}$ & $\checkmark$ & $\checkmark$ & $\checkmark$ & $\checkmark$ & - & - & - & $\checkmark$ & 62.5 & Moderate \\
\hline Kumar, $2017^{67}$ & $\checkmark$ & $\checkmark$ & $\checkmark$ & $\checkmark$ & $\checkmark$ & $\checkmark$ & $\checkmark$ & $\checkmark$ & 100 & Low \\
\hline Chaudhari et al., $2017^{68}$ & $\checkmark$ & $\checkmark$ & $\checkmark$ & $\checkmark$ & $\checkmark$ & $\checkmark$ & - & $\checkmark$ & 87.5 & Low \\
\hline Gouda and Rao, $2017^{69}$ & $\checkmark$ & $\checkmark$ & $\checkmark$ & $\checkmark$ & $\checkmark$ & $\checkmark$ & - & $\checkmark$ & 87.5 & Low \\
\hline Kapoor and Badyie, $2017^{70}$ & $\checkmark$ & $\checkmark$ & $\checkmark$ & $\checkmark$ & $\checkmark$ & $\checkmark$ & $\checkmark$ & $\checkmark$ & 100 & Low \\
\hline Naik et al., $2017^{71}$ & - & $\checkmark$ & $\checkmark$ & $\checkmark$ & $\checkmark$ & $\checkmark$ & - & $\checkmark$ & 75 & Low \\
\hline Sandhu et al., $2017^{72}$ & $\checkmark$ & $\checkmark$ & $\checkmark$ & $\checkmark$ & $\checkmark$ & $\checkmark$ & $\checkmark$ & $\checkmark$ & 100 & Low \\
\hline Tandon et al., $2017^{73}$ & - & $\checkmark$ & $\checkmark$ & $\checkmark$ & - & - & - & $\checkmark$ & 50 & Moderate \\
\hline Vignesh et al., $2017^{74}$ & $\checkmark$ & $\checkmark$ & $\checkmark$ & $\checkmark$ & $\checkmark$ & $\checkmark$ & $\checkmark$ & $\checkmark$ & 100 & \begin{tabular}{|l|} 
Low \\
\end{tabular} \\
\hline Ahmed et al., $2018^{2}$ & $\checkmark$ & $\checkmark$ & $\checkmark$ & $\checkmark$ & $\checkmark$ & $\checkmark$ & - & $\checkmark$ & 87.5 & Low \\
\hline Bai et al., $2018^{15}$ & $\checkmark$ & $\checkmark$ & $\checkmark$ & $\checkmark$ & $\checkmark$ & $\checkmark$ & $\checkmark$ & $\checkmark$ & 100 & Low \\
\hline
\end{tabular}




\begin{tabular}{|c|c|c|c|c|c|c|c|c|c|c|}
\hline Authors & \begin{tabular}{|l|l|} 
Q1 \\
\end{tabular} & Q2 & Q3 & Q4 & Q5 & Q6 & Q7 & Q8 & \% Yes & \begin{tabular}{|l|} 
Risk \\
\end{tabular} \\
\hline Herrera et al., $2018^{75}$ & $\checkmark$ & $\checkmark$ & $\checkmark$ & $\checkmark$ & $\checkmark$ & $\checkmark$ & $\checkmark$ & $\checkmark$ & 100 & Low \\
\hline Ishaq et al., $2018^{76}$ & - & $\checkmark$ & $\checkmark$ & $\checkmark$ & $\checkmark$ & $\checkmark$ & $\checkmark$ & $\checkmark$ & 87.5 & Low \\
\hline Manikya et al., $2018^{77}$ & $\checkmark$ & $\checkmark$ & $\checkmark$ & $\checkmark$ & $\checkmark$ & $\checkmark$ & $\checkmark$ & $\checkmark$ & 100 & Low \\
\hline Bhagyashree et al., $2018^{78}$ & - & - & $\checkmark$ & $\checkmark$ & - & - & $\checkmark$ & $\checkmark$ & 50 & Moderate \\
\hline Thomas et al., $2018^{79}$ & $\checkmark$ & $\checkmark$ & $\checkmark$ & $\checkmark$ & $\checkmark$ & $\checkmark$ & $\checkmark$ & $\checkmark$ & 100 & Low \\
\hline Divyadharsini and Kumar, $2019^{81}$ & $\checkmark$ & $\checkmark$ & $\checkmark$ & $\checkmark$ & $\checkmark$ & $\checkmark$ & $\checkmark$ & $\checkmark$ & 100 & Low \\
\hline Gurung et al., $2019^{82}$ & $\checkmark$ & $\checkmark$ & $\checkmark$ & $\checkmark$ & $\checkmark$ & $\checkmark$ & $\checkmark$ & $\checkmark$ & 100 & Low \\
\hline Vaishnavi et al., $2019^{83}$ & $\checkmark$ & $\checkmark$ & $\checkmark$ & $\checkmark$ & $\checkmark$ & $\checkmark$ & - & $\checkmark$ & 87.5 & Low \\
\hline Yendriwati et al., $2019^{84}$ & $\checkmark$ & $\checkmark$ & $\checkmark$ & $\checkmark$ & - & - & $\checkmark$ & $\checkmark$ & 75 & Low \\
\hline
\end{tabular}

Table 4. Risk of bias assessed by the Joanna Briggs Institute Critical Appraisal Tools for use in JBI Critical Appraisal Checklist for Analytical Cross Sectional Studies. Q1. Were the criteria for inclusion in the sample clearly defined? Q2. Were the study subjects and the setting described in detail? Q3. Was the exposure measured in a valid and reliable way? Q4. Were objective, standard criteria used for measurement of the condition? Q5. Were confounding factors identified? Q6. Were strategies to deal with confounding factors stated? Q7. Were the outcomes measured in a valid and reliable way? Q8. Was appropriate statistical analysis used? $\checkmark$ : Yes; -: No; U : Unclear: N/A: Not applicable.

Kaul et al. (2015)

Topczyiko et al. (Method 1) (2018)

Ramalingam et al. (2014)

Topczyiko et al. (Method 2) (2018)

Sharma ot al. (2014)

Nagalaxmi et al. (2014)

Topczyiko et al. (Method 3) (2018)

Sonal et al. (2005)

Bansal et al. (2019)

Overall $\left(p^{2}=97.71 \%, p<0.001\right)$

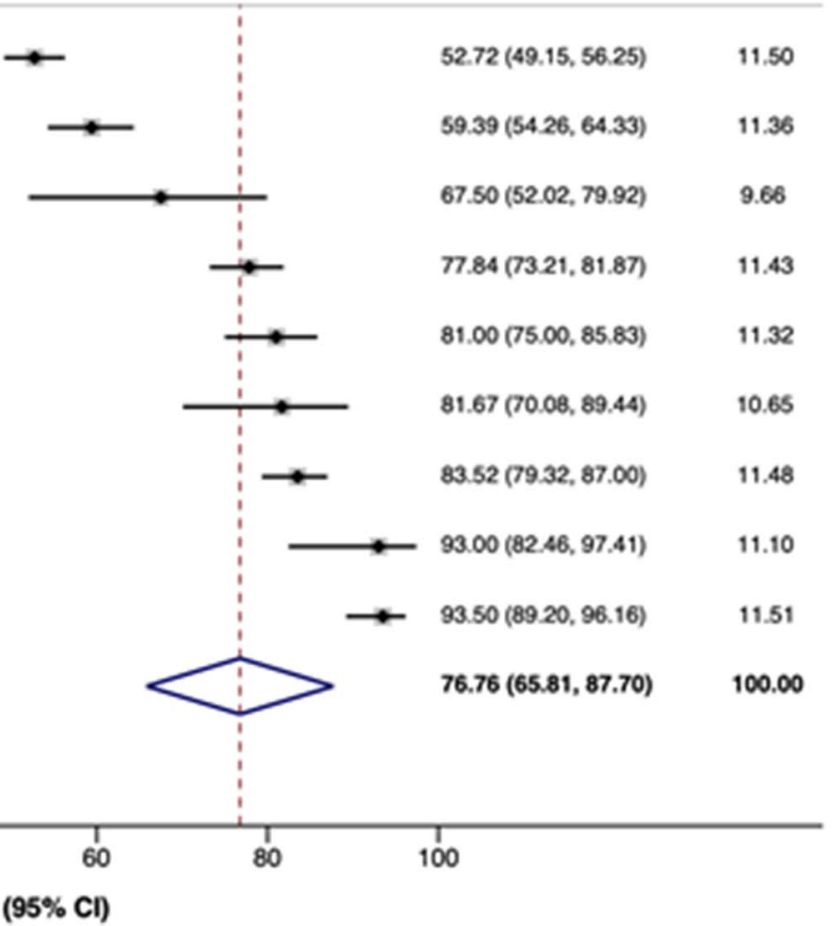

Figure 2. Overall compilation of accuracy rates across seven eligible studies that reported the sufficient data for quantitative analysis.

were already known, by other means, to have the diagnosis of interest and investigated whether the test of interest correctly identified them as such. Moreover, question 4 was marked as 'unclear' for three studies ${ }^{1,48,80}$ that did not provide details regarding blindness of the index test.

Synthesis of results. Primary outcome-accuracy for sexual dimorphism. Seven studies ${ }^{1,4,25,48,49,54,80}$ were included in the meta-analysis of the accuracy of lip prints for sexual dimorphism. Out of the seven studies, nine accuracy assessments were included in the meta-analysis- since the study by Topczyłko et al..$^{80}$ evaluated three different methods. The overall accuracy was $76.8 \%\left(95 \% \mathrm{CI}=65.8 ; 87.7, \mathrm{I}^{2}=97 \%\right)$ (Fig. 2). Individual accuracy rates ranged from 52.7 to $93.5 \%$.

Six out of the seven studies included in accuracy meta-analysis provided the number of hits and error according to the sex of the patient and were included in a meta-analysis that assessed if the odds of distinguishing males 


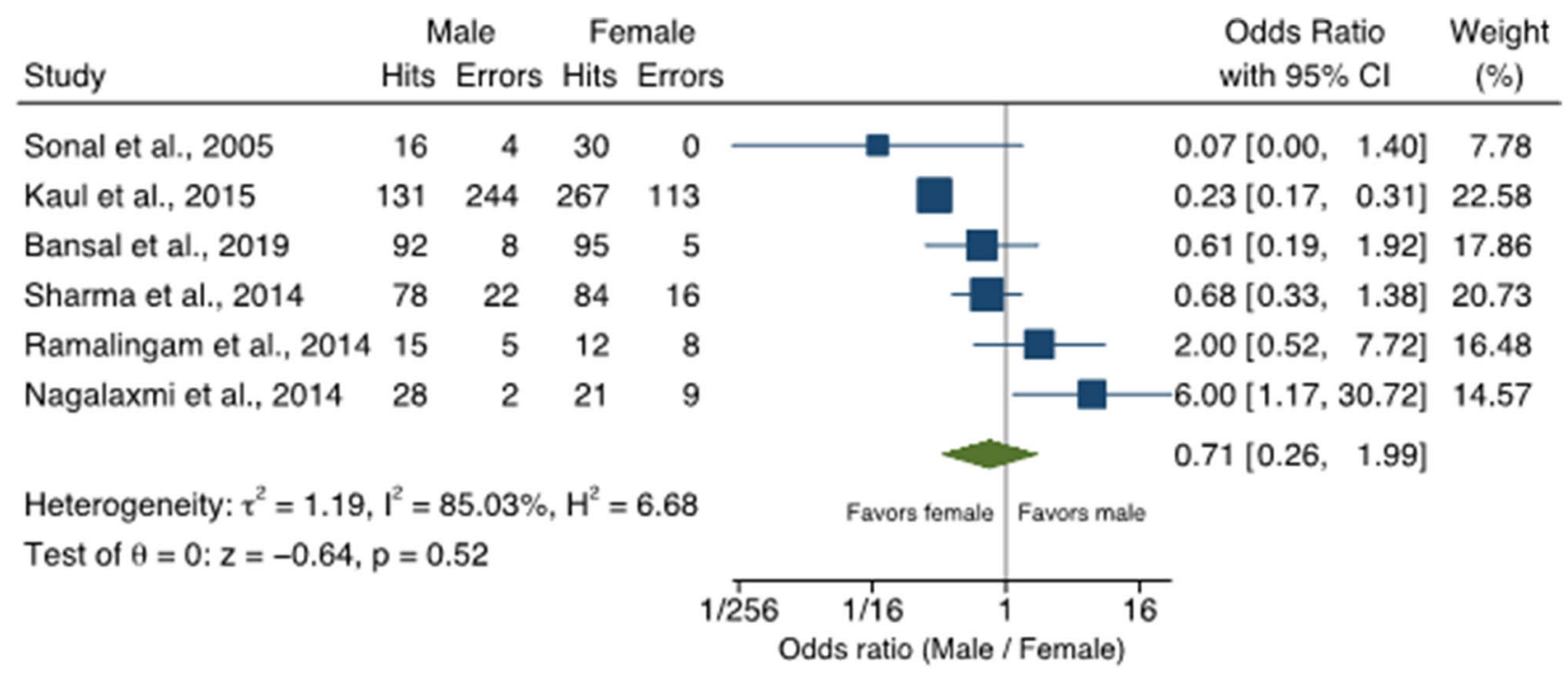

Random-effects model Sorted by: odds ratio

Figure 3. Odds ratio depicting the accuracy of cheiloscopy for distinguishing males from females. Randomeffects model applied within six eligible studies.

\begin{tabular}{|c|c|c|c|c|c|c|}
\hline & \multicolumn{3}{|c|}{$\begin{array}{l}\text { Left side using Suzuki and Tsuchihashi's } \\
(\mathrm{n}=14)^{9}\end{array}$} & \multicolumn{3}{|c|}{$\begin{array}{l}\text { Right side using Suzuki and Tsuchihashi's } \\
(\mathrm{n}=14)^{9}\end{array}$} \\
\hline & Male & Female & P value & Male & Female & P value \\
\hline \multicolumn{7}{|c|}{ Upper dental arch } \\
\hline Type 1 & $16.3(11.8 ; 21.4)$ & $16.0(11.2 ; 21.4)$ & 0.892 & $18.2(13.2 ; 23.7)$ & $17.2(11.7 ; 23.3)$ & 0.778 \\
\hline Type 1' & $12.4(6.6 ; 19.6)$ & $12.8(6.9 ; 20.0)$ & 0.964 & $12.6(6.7 ; 20.0)$ & $12.3(6.4 ; 19.9)$ & $0.928^{*}$ \\
\hline Type 2 & $23.7(20.9 ; 26.6)$ & $25.7(21.6 ; 30.0)$ & 0.473 & $23.7(20.9 ; 26.6)$ & $25.7(21.6 ; 30.0)$ & 0.473 \\
\hline Type 3 & $23.8(17.8 ; 30.4)$ & $18.0(11.1 ; 26.1)$ & 0.246 & $23.8(17.8 ; 30.4)$ & $18.0(11.1 ; 26.1)$ & 0.246 \\
\hline Type 4 & $10.2(6.7 ; 14.1)$ & $13.0(7.8 ; 19.4)$ & $0.454^{*}$ & $10.2(6.7 ; 14.1)$ & $13.0(7.6 ; 19.4)$ & $0.454^{*}$ \\
\hline Type 5 & $3.7(1.3 ; 6.9)$ & $3.5(1.1 ; 6.9)$ & 0.863 & $3.7(1.3 ; 6.9)$ & $3.5(1.1 ; 6.9)$ & 0.863 \\
\hline \multicolumn{7}{|c|}{ Lower dental arch } \\
\hline Type 1 & $19.7(10.4 ; 30.9)$ & $24.1(13.7 ; 36.3)$ & 0.580 & $23.7(14.1 ; 34.9)$ & $25.0(14.9 ; 36.8)$ & 0.875 \\
\hline Type 1' & $10.2(5.4 ; 16.3)$ & $11.3(6.3 ; 17.5)$ & 0.816 & $10.2(5.4 ; 16.3)$ & $11.3(6.3 ; 17.5)$ & $0.816^{*}$ \\
\hline Type 2 & $31.7(20.0 ; 44.7)$ & $31.4(22.3 ; 41.2)$ & 0.955 & $31.7(20.0 ; 44.7)$ & $31.4(22.3 ; 41.2)$ & 0.955 \\
\hline Type 3 & $18.2(8.5 ; 30.4)$ & $12.5(4.6 ; 23.3)$ & 0.435 & $18.2(8.5 ; 30.4)$ & $12.5(4.6 ; 23.3)$ & 0.435 \\
\hline Type 4 & $5.6(2.6 ; 9.4)$ & $5.2(1.9 ; 9.6)$ & 0.822 & $5.6(2.6 ; 9.4)$ & $5.2(1.9 ; 9.6)$ & $0.822^{*}$ \\
\hline Type 5 & $2.5(0.8 ; 4.9)$ & $1.9(0.6 ; 3.9)$ & 0.572 & $2.5(0.8 ; 4.9)$ & $1.9(0.6 ; 3.9)$ & 0.572 \\
\hline
\end{tabular}

Table 5. Lip pattern prevalence according to sex and dental arch for Suzuki and Tsuchihashi's method for cheiloscopy classification. ${ }^{\star}$ Evidence of publication bias according to Egger's test $(p<0.05)$. 'Evidence from 14 studies.

was different compared to the odds of distinguishing females. Overall, there were no differences to diagnose males compared to females $\left(\mathrm{OR}=0.71 ; 95 \% \mathrm{CI}=0.26 ; 1.99, \mathrm{I}^{2}=85 \%\right)$. Only specific studies, such as Kaul et al. $(2015)^{53}$ and Nagalaxmi et al. (2014) $)^{48}$, described differences for sexual dimorphism (Fig. 3). The first showed $77 \%$ higher odds of identifying females compared to males $(\mathrm{OR}=0.23 ; 95 \% \mathrm{CI}=0.27 ; 0.31)$, while the second showed sixfold higher odds of identifying males compared to females $(\mathrm{OR}=6.00 ; 95 \% \mathrm{CI}=1.17 ; 30.72)$. One study ${ }^{80}$ did not report samples divided by sex and was not included in the analysis.

Secondary outcome-prevalence of lip prints. According to the technique of Suzuki and Tsuchihashi (1970), lip print pattern type 2 was the most prevalent $(>30 \%)$, while type 5 was the rarest pattern $(<3 \%)$ (Table 5$)$. Sex differences based on prevalence rates were not detected. Publication bias was identified for studies analyzing lip print type 1' for the upper and lower dental arches on the right side, for lip print type 4 for the upper arch on the left and right sides, and for lip print type 4 for the lower arch on the right side.

Sex differences were not observed using Renaud's (1970) technique. According to this technique, the most prevalent pattern was type C (>12\%), while type I was the least prevalent $(<1 \%)$ (Table 6$)$. 


\begin{tabular}{|c|c|c|c|c|c|c|}
\hline & \multicolumn{3}{|c|}{ Left side using Renaud's $(\mathrm{n}=3)^{9}$} & \multicolumn{3}{|c|}{ Right side using Renaud's $(\mathrm{n}=3)^{9}$} \\
\hline & Male & Female & P value & Male & Female & P value \\
\hline \multicolumn{7}{|c|}{ Upper dental arch } \\
\hline Type A & $12.7(3.2 ; 26.9)$ & $8.1(0.2 ; 25.0)$ & 0.622 & $8.0(0.0 ; 29.5)$ & $6.7(0.0 ; 23.7)$ & 0.889 \\
\hline Type B & $8.5(0.0 ; 29.7)$ & $6.8(0.0 ; 25.0)$ & 0.867 & $12.2(0.0 ; 42.0)$ & $8.3(0.0 ; 30.0)$ & 0.783 \\
\hline Type C & $12.6(3.2 ; 26.8)$ & $18.8(10.6 ; 28.7)$ & 0.439 & $12.4(0.8 ; 34.2)$ & $19.5(8.2 ; 34.2)$ & 0.542 \\
\hline Type D & $5.2(2.4 ; 8.9)$ & $5.4(1.1 ; 12.6)$ & 0.952 & $6.9(0.5 ; 19.4)$ & $7.5(0.9 ; 19.7)$ & 0.922 \\
\hline Type E & $8.0(1.6 ; 18.6)$ & $9.4(4.3 ; 16.4)$ & 0.796 & $9.6(2.8 ; 19.8)$ & $4.8(0.2 ; 14.2)$ & 0.399 \\
\hline Type F & $2.2(0.0 ; 10.4)$ & $2.5(0.0 ; 8.7)$ & 0.937 & $1.3(0.0 ; 5.3)$ & $1.8(0.0 ; 6.6)$ & 0.840 \\
\hline Type G & $15.3(4.6 ; 30.6)$ & $8.9(2.9 ; 17.8)$ & 0.399 & $7.3(0.6 ; 20.3)$ & $8.3(1.9 ; 18.5)$ & 0.892 \\
\hline Type $\mathrm{H}$ & $11.3(2.4 ; 25.2)$ & $11.5(0.7 ; 32.2)$ & 0.979 & $11.9(1.5 ; 30.1)$ & $12.0(0.9 ; 32.7)$ & 0.999 \\
\hline Type I & $0.0(0.0 ; 0.3)$ & $0.8(0.0 ; 3.1)$ & 0.166 & $0.0(0.0 ; 0.3)$ & $0.8(0.1 ; 2.0)$ & 0.048 \\
\hline Type J & $9.0(0.0 ; 34.2)$ & $14.2(0.1 ; 44.2)$ & 0.736 & $13.0(4.3 ; 25.3)$ & $10.9(0.0 ; 38.9)$ & 0.867 \\
\hline \multicolumn{7}{|c|}{ Lower dental arch } \\
\hline Type A & $4.8(0.0 ; 21.4)$ & $4.8(0.1 ; 24.8)$ & 0.998 & $9.7(1.5 ; 23.8)$ & $5.8(0.0 ; 25.2)$ & 0.678 \\
\hline Type B & $5.6(0.0 ; 24.5)$ & $9.0(0.0 ; 29.7)$ & 0.739 & $3.0(00 ; 19.1)$ & $6.7(0.0 ; 26.9)$ & 0.664 \\
\hline Type C & $17.8(5.0 ; 36.4)$ & $22.5(5.2 ; 47.1)$ & 0.744 & $19.3(5.3 ; 39.2)$ & $27.0(7.8 ; 52.8)$ & 0.603 \\
\hline Type D & $8.0(5.0 ; 11.7)$ & $6.2(3.9 ; 8.9)$ & 0.375 & $8.8(3.8 ; 15.6)$ & $5.2(3.8 ; 6.7)$ & 0.201 \\
\hline Type E & $15.0(3.2 ; 33.1)$ & $18.3(6.3 ; 34.8)$ & 0.762 & $16.7(3.5 ; 36.8)$ & $17.9(5.6 ; 35.0)$ & 0.921 \\
\hline Type F & $4.9(0.0 ; 24.6)$ & $3.7(0.0 ; 13.5)$ & 0.868 & $2.1(0.0 ; 7.9)$ & $2.6(0.0 ; 9.3)$ & 0.884 \\
\hline Type G & $12.2(4.6 ; 22.8)$ & $8.9(3.1 ; 17.3)$ & 0.573 & $12.2(5.2 ; 21.5)$ & $8.8(2.6 ; 17.9)$ & 0.556 \\
\hline Type $\mathrm{H}$ & $8.2(0.3 ; 24.4)$ & $7.5(0.4 ; 21.8)$ & 0.930 & $6.6(1.2 ; 15.7)$ & $7.9(0.1 ; 24.9)$ & 0.867 \\
\hline Type I & $0.1(0.0 ; 0.8)$ & $0.1(0.0 ; 0.7)$ & 0.967 & $0.0(0.0 ; 0.2)$ & $0.3(0.0 ; 1.7)$ & 0.377 \\
\hline Type J & $4.5(0.0 ; 15.4)$ & $4.6(0.6 ; 11.8)$ & 0.991 & $8.6(5.2 ; 12.8)$ & $4.9(0.7 ; 12.2)$ & 0.329 \\
\hline
\end{tabular}

Table 6. Lip pattern prevalence according to sex and dental arch for Renaud's method for cheiloscopy classification. "Evidence from 14 studies.

\begin{tabular}{|c|c|c|c|c|c|c|c|c|c|c|}
\hline \multicolumn{7}{|c|}{ Quality assessment } & \multicolumn{3}{|c|}{ Summary of results } & \multirow[b]{3}{*}{ Importance } \\
\hline \multirow[b]{2}{*}{$\mathbf{N}$} & \multirow[b]{2}{*}{ Study Design } & \multirow[b]{2}{*}{$\begin{array}{l}\text { Methodological } \\
\text { Limitations }\end{array}$} & \multirow[b]{2}{*}{ Inconsistency } & \multirow[b]{2}{*}{ Indirectness } & \multirow[b]{2}{*}{ Imprecision } & \multirow[b]{2}{*}{$\begin{array}{l}\text { Other } \\
\text { considerations }\end{array}$} & \multirow[b]{2}{*}{$\begin{array}{l}\text { Number of } \\
\text { participants }\end{array}$} & \multirow{2}{*}{$\begin{array}{l}\text { Effect } \\
\text { Accuracy (95\% } \\
\text { CI) }\end{array}$} & \multirow[b]{2}{*}{ General quality } & \\
\hline & & & & & & & & & & \\
\hline \multicolumn{11}{|c|}{ "Is cheilososcopy a reliable method for estimating sex?" } \\
\hline 7 & $\begin{array}{l}\text { Diagnostic accu- } \\
\text { racy studies }\end{array}$ & Not serious ${ }^{\mathrm{a}}$ & Serious $^{\mathrm{b}}$ & Not serious $^{c}$ & Serious $^{\mathrm{d}}$ & none & 1547 & $\begin{array}{l}76.76(65.81- \\
87.70)\end{array}$ & $\oplus \oplus \mathrm{LOW}$ & Critical \\
\hline
\end{tabular}

Table 7. Grading of Recommendations Assessment, Development, and Evaluation (GRADE) summary of findings table for the outcomes of the systematic review and meta-analysis. GRADE Working Group grades of evidence. High certainty: We are very confident that the true effect lies close to that of the estimate of the effect. Moderate certainty: We are moderately confident in the effect estimate: The true effect is likely to be close to the estimate of the effect, but there is a possibility that it is substantially different. Low certainty: Our confidence in the effect estimate is limited: The true effect may be substantially different from the estimate of the effect. Very low certainty: We have very little confidence in the effect estimate: The true effect is likely to be substantially different from the estimate of effect. ${ }^{a}$ Majority of the studies presented low risk of bias; ${ }^{\mathrm{b}}$ The heterogeneity $\left(\mathrm{I}^{2}\right)$ was high $(>75 \%)$ and no overlapping of effect estimates; ${ }^{c}$ Evidence stems from an adequate population; ${ }^{\mathrm{d}}$ Wide credible confidence interval.

Certainty of evidence. GRADE approach showed low certainty of evidence. The limiting aspects were the lack of consistency between the estimated effects and the lack of overlap of confidence intervals-evidenced by the increased heterogeneity between the included studies (Table 7).

\section{Discussion}

Dental analysis, within forensic dentistry, figures as an alternative for human identification especially because of the resistance of human teeth to high temperature and cadaveric alterations ${ }^{85}$. Over time, several forensic applications were studied for the use of dental/oral evidence. Apart human identification, bite mark analysis ${ }^{86}$ anthropological estimation of age ${ }^{87}, \operatorname{sex}^{88}$, stature ${ }^{89}$ and ancestry ${ }^{90}$; rugoscopy ${ }^{91}$ and cheiloscopy ${ }^{92}$ currently represent fields of forensic odontology. While some fields developed with strong scientific basis and broad legal acceptance (i.e. human identification), other fields remained controversial and lacked high-level evidence-based confirmation-this is the case of cheiloscopy. From the perspective of forensic practice, the alleged contribution 
of cheiloscopy relies on the possibility of retrieving identifying information (such as sex) from a suspect from visible or latent lip prints left in a crime scene ${ }^{93}$. Two main controversies might arise from cheiloscopy: (I) in crime scene investigations, the existing lip print left on objects or other surfaces could enable higher evidence toward human identification through DNA extraction instead of comparative analysis of furrows; (II) studies on cheiloscopy are generally observational, cross-sectional and with questionable settings that include different techniques, underlying surfaces and registration materials (e.g. lipsticks and powdered metals). In this scenario, several questions are pertinent: Why the scientific literature is so vast of studies on cheiloscopy for sexual dimorphism? How often is cheiloscopy used by forensic dentists in practice? But especially (claimed in many studies): Is cheiloscopy really useful to distinguish male and females in forensic dentistry?

To the present, there is no antemortem database of lip patterns worldwide (even in clinical dentistry). Moreover, registering the lips with photographs or other tools is rare-so, the application of cheiloscopy for human identification is limited from the beginning. Striving for sexual dimorphism could be an interesting asset to the armamentarium of forensic dentists, but again the application in practice is relative, especially because dental human identification is mainly necessary in challenging cases that involve charred bodies and skeletal remains ${ }^{94}$-in which lips are usually destroyed. Additionally, sexual dimorphism should be accomplished from body structures scientifically known for their anthropological reliability, namely the pelvic bones and skull ${ }^{95}$.

The evidence brought through the present systematic review was extracted from 72 studies that sampled 22,965 individuals. Out of the studies, $70 \%(n=52)^{1,4-6,10-15,25,27,30,31,35-40,43-46,48-50,53-57,59-62,64,66-75,78,79,81,83,84}$ were from India. At first sight, the quality of studies was not bad when it comes to assessment of the risk of bias (nearly $70 \%$ had low risk of bias). These outcomes combined with the general quantification of the studies that detected sex differences based on lip pattern (67\%) could lead to dangerous interpretations from readers that are not familiar with systematic reviews. A deeper look on the quantified outcomes of the most prevalent techniques (Suzuki \& Tsuchihashi, 1970, $\mathrm{n}=64,88 \%$; Renaud et al., 1973, $\mathrm{n}=4,5 \%$ ), however, depicts an emerging lack of statistical significance $(\mathrm{p}>0.05)$ for each lip pattern between males and females. The analysis performed per pattern clarifies the scenario as most of the studies in the field only test sexual dimorphism by comparing generalized (combined) patterns within sex groups (males vs. females). Further on, the limitations of cheiloscopy for sexual dimorphism is corroborated by GRADE assessment outcomes, which pooled seven studies $(10 \%$ of selected studies) and 1,547 participants to clearly point out high heterogeneity ( $>75 \%)$. The heterogeneity might be justified mainly because none of the 72 observational eligible studies reported data using scientifically established guidelines, namely STROBE. The resulting analysis via GRADE suggested low level of general quality and critical level of importance. Considering the diagnostic accuracy of cheiloscopy, mean outcomes point to $76 \%$, which indicates that one in every four analysis of sexual dimorphism through lip patterns will have a wrong classification. Stronger outcomes would necessarily require a higher level of accuracy and a lower level of heterogeneity across studies. Summed up, the eligible studies screened and assessed in the present systematic review showed a good performance of cheiloscopy when the studies were analyzed separately; but when it comes to deeper analyses, especially observed per lip pattern within the techniques, lack of evident differences were detected between males and females. The limitation of cheiloscopy is, therefore, corroborated with the final quantitative assessment via GRADE.

To the present, the alleged contribution of cheiloscopy in forensic dentistry is merely superficial and highly relative. The quantification of the potential error within the diagnostic accuracy of cheiloscopy would be close to $25 \%$-in other words, nearly 386 participants sampled in the quantitative part of this review would have their sex wrongly classified from a sample of 1547 individuals. Forensic dentistry itself is already a relative tool for human identification (not necessarily applicable in every single autopsy). In general, charred victims and skeletal remains consist of the main scenarios for a forensic odontologist. Authors might claim lip print applications to narrow disaster victim identification lists by sex, but in most of these cases bodies are not intact. If the case is somehow improving cheiloscopy studies in the future, authors are encouraged to design more advanced analyses of the morphology of the human lips to the point of having enough evidence to support the development of clinical databases and protocols for lip recording. From the perspective of forensic practice, this systematic review does not encourage the use of cheiloscopy as the sole tool for sexual dimorphism.

\section{Conclusion}

After revisiting 72 eligible studies with a pooled sample of 22,965 individuals, this systematic review revealed weak foundations for the use of lip print analysis for sexual dimorphism in forensic dentistry. The pooled sampled reduced within the meta-analysis showed an average rate of wrong sex classification of nearly $25 \%$. The studies were highly heterogeneous as none of them followed proper EQUATOR guidelines for structuring methods and reporting data. GRADE analysis confirmed the low certainty of evidence suggesting that cheiloscopy is not a reliable tool in practice when it comes to sexual dimorphism.

\section{Data availability}

The datasets generated during and/or analysed during the current study are available from the corresponding author on reasonable request.

Received: 11 March 2021; Accepted: 1 December 2021

Published online: 17 December 2021

\section{References}

1. Bansal, A. K., Doshi, S., Bansal, P., Patel, R. \& Barai, P. H. Cheiloscopy: A lip print study. Indian J. Med. Forensic Med. Toxicol. 13, 35-39 (2019). 
2. Ahmed, S. A., Salem, H. E. \& Fawzy, M. M. Forensic dissection of lip print as an investigative tool in a mixed Egyptian population. Alex. Med. J. 54, 235-239 (2018).

3. Adamu, L. H. \& Taura, M. G. Lip prints: An emerging tool for personal identification. J. Biomed. Sci. 1, 78-87 (2016).

4. Alzapur, A., Nagothu, R. S. \& Nalluri, H. B. Lip prints-A study of its uniqueness among students of MediCiti Medical College. Indian J. Clin. Anat. Physiol. 4, 68-70 (2017).

5. Sharma, V., Ingle, N. A., Kaur, N. \& Yadav, P. Identification of sex using lip prints: A clinical study. J. Int. Soc. Prev. Commun. 4, $173-177$ (2014).

6. Bansal, N., Sheikh, S., Bansal, R. \& Pallagati, S. Correlation between lip prints and finger prints in sex determination and pattern predominance in 5000 subjects. J. Forensic Odontostomatol. 3, 8-14 (2013).

7. Franco, A., Thevissen, P., Fieuws, S., Souza, P. H. C. \& Willems, G. Applicability of Willems model for dental age estimations in Brazilian children. Forensic Sci. Int. 231, 1-4 (2013).

8. International Criminal Police Organization. Disaster victim identification guide. Available from: https://www.interpol.int/Howwe-work/Forensics/Disaster-Victim-Identification-DVI (2018)

9. Ramakrishnan, P., Bahirwani, S. \& Valambath, S. Assessment of cheiloscopy in sex determination using lysochrome-A preliminary study. J. Forensic Dent. Sci. 7, 195-200 (2015).

10. Augustine, J., Barpande, S. R. \& Tupkari, J. V. Cheiloscopy as an adjunct to forensic identification: A study of 600 individuals. J. Forensic Odontostomatol. 26, 44-52 (2008).

11. Amith, H. V., Ankola, A. V. \& Nagesh, L. Cheiloscopic comparison of the tibetan refugees in mundgod and the population of Belgaum, India. Indian J. Med. Forensic Med. Toxicol. 6, 9-12 (2012).

12. Babladi, P. I., Satish, B. N. V., Raghavendra, S. K. M., Uzair, S. H. \& Reddy, M. Lip Prints-effective tool of identification and sex determination. Indian J. Med. Forensic Med. Toxicol. 6, 74-75 (2012).

13. Abidullah, M., Kumar, M. N., Bhorgonde, K. D. \& Reddy, D. S. P. Cheiloscopy and dactyloscopy: Do they dictate personality patterns?. J. Forensic Dent. Sci. 7, 114-1120 (2015).

14. Basheer, S., Gopinath, D., Shameena, P. M., Sudha, S. \& Lakshmi, J. D. Correlation of lip patterns, gender, and blood group in North Kerala population: A study of over 800 individuals. J. Forensic Dent. Sci. 9, 73-77 (2017).

15. Bai, J. K. S. et al. Correlative study on lip prints, fingerprints, and mandibular intercanine distance for gender determination. J. Forensic. Dent. Sci. 10, 143 (2018).

16. Page, M.J., McKenzie, J., Bossuyt, P., Boutron, I., Hoffmann, T., Mulrow, C.D., Moher, D. The PRISMA 2020 statement: an updated guideline for reporting systematic reviews (2020).

17. McInnes, M. D. F., Moher, D. \& Thombs, B. D. Preferred reporting items for a systematic review and meta-analysis of diagnostic test accuracy studies: The PRISMA-DTA statement. JAMA 319, 388-396 (2018).

18. Aromataris, E. \& Munn, Z. (eds) Joanna Briggs Institute Reviewer's Manual 4th edn. (The Joanna Briggs Institute, Adelaide, 2017).

19. Moola, S. et al. Methodological quality of case series studies: An introduction to the JBI critical appraisal tool. JBI Evid. Synth. 18, 2127-2133 (2020)

20. Campbell, J. M. et al. Diagnostic test accuracy: Methods for systematic review and meta-analysis. Int. J. Evid. Based Healthc. 13, 154-162 (2015).

21. Freeman, M. F. \& Tukey, J. W. Transformations related to the angular and the square root. Ann. Math. Statist. 21, 607-611 (1950).

22. Hunter, J. P. et al. In meta-analyses of proportion studies, funnel plots were found to be an inaccurate method of assessing publication bias. J. Clin. Epidemiol. 67, 897-903 (2014).

23. Balshem, H. et al. GRADE guidelines: 3. Rating the quality of evidence. J. Clin. Epidemiol. 64, 401-406 (2011).

24. Fauvel, L. F. Etude des empreintes labiales par une méthode originale d'enregistrement au silicium organique. Larc. Med. 2, 465-471 (1982) ((in French)).

25. Sonal, V. \& Nayak, P. Study of lip-prints as aid for sex determination. Med. Legal Update. 5, 93-98 (2005).

26. Barros, G. B. Cheiloscopy: Use of the Technique in the Forensic Identification (University of São Paulo, 2006).

27. Sharma, P., Saxena, S. \& Rathod, V. Comparative reliability of cheiloscopy and palatoscopy in human identification. Indian J. Dent. Res. 20, 453-457 (2009).

28. El Domiaty, M. A., Al-gaidi, S. A., Elayat, A. A., Safwat, M. D. \& Galal, S. A. Morphological patterns of lip prints in Saudi Arabia at Almadinah Almonawarah province. Forensic Sci. Int. 200(179), e1-179.e1799 (2010).

29. Chalapud, D., Mosquera, M. F., Pulgarín, M. F., Cruz, C. \& Moreno, F. Análisis queiloscópico en estudiantes de odontología de la ciudad de Cali. Estomatología. 19, 14-20 (2011).

30. Gupta, S., Gupta, K. \& Gupta, O. P. A study of morphological patterns of lip prints in relation to gender of North Indian population. J. Oral. Biol. Craniofac. Res. 1, 12-16 (2011).

31. Prasad, P. \& Vanishree, V. A. Comparison of lip prints between Aryans-Dravidians and Mongols. Indian J. Dent. Res. 22, 664-668 (2011)

32. Costa, V. A. \& Caldas, I. M. Morphologic patterns of lip prints in a Portuguese population: a preliminary analysis. J. Forensic Sci. 57, 1318-1322 (2012).

33. Karki, R. K. Lip prints—An identification aid. Kathmandu Univ. Med J. 10, 55-57 (2012).

34. Oliveira, J. A., Rabello, P. M. \& Fernandes, L. C. C. Estudo Queiloscópico em Graduandos de Odontologia. Pesqui Bras. Odontopediatria Clín. Integr. 12, 521-528 (2012).

35. Prabhu, R. V., Ajit, D. \& Prabhu, V. A study of lip print pattern in Goan dental students-A digital approach. J. Forensic Leg. Med. 19, 390-395 (2012).

36. Rastogi, P. \& Amrita, P. Lip prints-An aid in identification. Aust. J. Forensic Sci. 44, 109-116 (2012).

37. Vats, Y., Jasmine, K. D. \& Kapoor, A. K. Gender variation in morphological patterns of lip prints among some north Indian Populations. J. Forensic Dent. Sci. 4, 19-23 (2012).

38. Kautilya, V., Pravir, B. D. \& Naveen, R. Efficacy of cheiloscopy in determination of sex among South Indians. J. Clin. Diagn. Res. 7, 2193-2196 (2013)

39. Koneru, A. et al. Comparison of lip prints in two different populations of India: Reflections based on a preliminary examination. J. Forensic Dent. Sci. 5, 11-15 (2013).

40. Padmavathi, B. N., Makkad, R. S., Rajan, S. Y. \& Kolli, G. K. Gender determination using cheiloscopy. J. Forensic Dent. Sci. 5, 123-128 (2013).

41. Popa, M. F., Corina, S. \& Paul-Daniel, C. Medical-legal identification methods with the aid of cheiloscopy. Rom. J. Legal Med. 21, 215-218 (2013)

42. Ragab, A. R., Sahar, A. E., El-Dakroory, S. A. E. \& Rania, H. A. R. Characteristic patterns of lip prints in Egyptian population sample at Dakahlia Governorate. J. Legal Med. 127, 521-527 (2013).

43. Sekhon, J., Singla, R., Sharma, T., Tayal, I. \& Singh, A. Most predominating pattern of lip prints in north Indian population. J. Indian Acad. Forensic Med. 35, 362-366 (2013).

44. Verma, P., Sachdeva, S. K., Verma, K. G., Saharan, S. \& Sachdeva, K. Correlation of lip impressions with gender, ABO blood groups intercomissural distance. North Am. J. Med. Sci. 5, 427-431 (2013).

45. Gupta, S., Gupt, K., Gupta, O. P. \& Verma, A. K. Evaluation of the degree of agreement in identifying lip prints and palatal rugae by three independent observers and valuation of there dependability in sex determination. Indian J. Med. Forensic Med. Toxicol. 8, 214-220 (2014). 
46. Hammad, M., Habib, H. \& Bhatti, Y. A. Gender variation of lip prints among the students of Avicenna Medical College, Lahore. PaK. J. Med. Sci. 8, 321-324 (2014).

47. Multani, S., Thombre, V., Thombre, A. \& Surana, P. Assessment of lip print patterns and its use for personal identification among the populations of Rajnandgaon, Chhattisgarh, India. J. Int. Soc. Prev. Commun. 4, 170-174 (2014).

48. Nagalaxmi, V. et al. Cheiloscopy, palatoscopy and odontometrics in sex prediction and dis-crimination-a comparative study. Open Dent. J. 8, 269-279 (2014).

49. Ramalingam, K., Misra, N., Deepak, U. \& Misra, P. Cheiloscopy for sex determination: a study. Univers. Res. J. Dent. 14, 48-51 (2014).

50. Badiye, A. \& Kapoor, N. Morphologic variations of lip-print patterns in a Central Indian population: A preliminary study. Med. Sci. Law. 56, 200-204 (2016).

51. Bharathi, S. \& Thenmozhi, M. S. Cheiloscopy-Lip print, an determination of sex and individual. Int. J. Pharm. Sci. Res. 7, 330-333 (2015).

52. Cartaxo, L. L. Identificação médico-legal de uma população Portuguesa através da queiloscopia (University of Lisbon, 2015).

53. Hernández, J. C. M., Yuli, N. O. P. \& Paredes, J. F. M. Identificación de sexo mediante queiloscopia en Santander, Colombia: una herramienta para la medicina forense: estudio inicial. Rev. Esp. Med. Legal. 41, 111-116 (2015) ((in Spanish)).

54. Kaul, R., Padmashree, S. M., Shilpa, P. S., Sultana, N. \& Bhat, S. Cheiloscopic patterns in Indian population and their efficacy in sex determination: A randomized cross-sectional study. J. Forensic Dent. Sci. 7, 101-106 (2015).

55. Nagpal, B., Hegde, U., Sreeshyla, H. S. \& Arun, M. Comparative evaluation of lip prints among Indian and Malaysian students. J. Indian Forensic Sci. 37, 131-134 (2015).

56. Peeran, S. W. et al. A study of lip print patterns among adults of Sebha city, Libya. J. Forensic Dent. Sci. 7, 67-70 (2015).

57. Shah, K. K. \& Jayaraj, G. Cheiloscopy for sex determination among individuals aged 17-25 years. Int. J. Pharm. Sci. Res. 7, 731-735 (2015).

58. Sharma, R., Sharma, K., Preethi, N., Degra, H. \& Rajmani, H. Cheiloscopy: a study of morphological patterns of lip prints in Rajasthani population. J. Res. Med. Dent. Sci. 3, 35-38 (2015).

59. Aziz, M. H. A., El Dine, F. M. M. B. \& Saeed, N. M. M. Regression equations for sex and population detection using the lip print pattern among Egyptian and Malaysian adult. J. Forensic Legal Med. 44, 103-110 (2016).

60. Borase, A. P., Saleem, S. \& Anand, M. A study of lip prints among North Maharashtrian population. J. Adv. Oral Res. 7, 20-25 (2016).

61. Jeergal, P. A., Pandit, S., Desai, D., Surekha, R. \& Jeergal, V. A. Morphological patterns of lip prints in Mangaloreans based on Suzuki and Tsuchihashi classification. J. Oral Maxillofac. Surg. Med. Pathol. 20, 320 (2016).

62. Krishnan, R. P., Thangavelu, R., Rathnavelu, V. \& Narasimhan, M. Gender determination: role of lip prints, fingerprints, and mandibular canine index. Exp. Ther. Med. 11, 2329-2332 (2016).

63. Moshfeghi, M., Beglou, A., Mortazavi, H. \& Bahrololumi, N. Morphological patterns of lip prints in an Iranian population. J. Clin. Exp. Dent. 8, 550-555 (2016).

64. Negi, A. \& Negi, A. The connecting link! Lip prints and fingerprints. J. Forensic Dent. Sci. 8, 177-181 (2016).

65. Simovic, M., Pavušek, I., Muhasilović, S. \& Vodanović, M. Morphologic patterns of lip prints in a sample of Croatian population. Acta Stomatol. Croat. 50, 122-127 (2016).

66. Tarvadi, P. V. \& Goyal, A. K. Cheiloscopy-Role in forensic investigations. J. Punjab Acad. Forensic Med. Toxicol. 16, 23-26 (2016).

67. Kumar, E. D. B. Study of lip prints as a tool for identification: an assessment on 200 Subjects in South India. Indian J. Med. Forensic Med. Toxicol. 11, 31 (2017).

68. Chaudhari, S. H. et al. A study of cheiloscopic patterns in Chhattisgarh population. Indian J. Med. Forensic Med. Toxicol. 11, 119-123 (2017).

69. Gouda, S. \& Rao, M. R. Morphological study of lip print pattern among medical students: an anthropological study. Medico-Legal Update. 17, 213-216 (2017).

70. Kapoor, N. \& Badiye, A. A study of distribution, sex differences and stability of lip print patterns in an Indian population. Saudi J. Biol. Sci. 24, 1149-1154 (2017).

71. Naik, R., Mujib, B. A., Telagi, N. \& Hallur, J. Comparative analysis of lip with thumbprints: An identification tool in personal authentication. J. Oral Maxillofac. Pathol. 21, 171-175 (2017).

72. Sandhu, H., Verma, P., Padda, S. \& Sunder, R. S. Frequency and correlation of lip prints, fingerprints and ABO blood groups in population of Sriganganagar District, Rajasthan. Acta Med. Acad. 46, 105-115 (2017).

73. Tandon, S. A., Jaiswal, R., Patidar, M. \& Khare, A. Estimation of gender using cheiloscopy and dermatoglyphics. Natl. J. Maxillofac. Surg. 8, 102-105 (2017).

74. Vignesh, R., Rekha, C. V., Annamalai, S., Norouzi, P. \& Sharmin, D. Comparative evaluation between cheiloscopic patterns and terminal planes in primary dentition. Contemp. Clin. Dent. 8, 522-525 (2017).

75. Herrera, L. M., Clemente, M. S. F. \& Monica, C. S. Evaluation of lip prints on different supports using a batch image processing algorithm and image superimposition. J. Forensic Sci. 63, 122-129 (2018).

76. Ishaq, N., Malik, A. R., Ahmad, Z. \& Ullah, S. E. Determination of sex by cheiloscopy as an aid to establish personal identity. Ann. King Edw. Med. Univ. 24, 581-585 (2018).

77. Manikya, S. et al. Comparison of Cheiloscopy and Rugoscopy in Karnataka, Kerala, and Manipuri Population. J. Int. Soc. Prev. Commun. Dent. 8, 439-445 (2018).

78. Bhagyashree, B. et al. Sex determination using cheiloscopy and mandibular canine index as a tool in forensic dentistry. J. Forensic Legal Med. 4, 23-30 (2018).

79. Thomas, A. J. et al. Morphology of lip print patterns among Indian and Malaysian population-A tool for racial and gender identification. Indian J. Med. Forensic Med. Toxicol. 12, 272-277 (2018).

80. Topczyłko, A., Borysławski, K. \& Nowakowski, D. A comparison of sex identification methods based on lip furrow pattern. Anthropol. Rev. 81, 45-52 (2018).

81. Divyadharsini, V. \& Kumar, J. V. Analysing cheiloscopic pattern and mandibular canine index for gender determination. Int. J. Pharm. Res. 2, 254-258 (2019).

82. Gurung, S., Vijay, G. \& Lamichhane, A. Prevalence of type I lip print among medical students in a medical college of Nepal. J. Nepal Med. Assoc. 57, 221-225 (2019).

83. Vaishnavi, A., Sangeetha, S. \& Premavathy, D. Lip prints-A study of its uniqueness, prevalence, and gender significance. Drug Inv. Today 12, 781-784 (2019).

84. Yendriwati, Y., Joe, J. W. \& Fitri, A. R. Lip printing as a method of forensic identification in ethnic Malays Chinese. J. Evol. Med. Dent. Sci. 8, 2-11 (2019).

85. Selvarason, T. Significance of restorations in forensic identification. J. Forensic Dent. Sci. 6, 207-209 (2014).

86. Franco, A. et al. Three-dimensional analysis of the uniqueness of the anterior dentition in orthodontically treated patients and twins. Forensic Sci. Int. 273, 80-87 (2017).

87. Willems, G., Van Olmen, A., Spiessens, B. \& Carels, C. Dental age estimation in Belgian children: Demirjian's technique revisited. J. Forensic Sci. 46, 893-895 (2001).

88. Capitaneanu, C., Willems, G. \& Thevissen, P. Evidence-based mapping of third molar techniques for age estimation applied to Brazilian adolescents-A systematic review. J. Forensic Odontostomatol. 35, 1-19 (2017). 
89. Hossaim, M. Z., Munawar, K. M. M., Rahim, Z. H. A. \& Bakri, M. M. Can stature be estimated from tooth crown dimensions? A study in a sample of South-East Asians. Arch. Oral Biol. 64, 85-91 (2016).

90. Edgar, H. J. H. Estimation of ancestry using dental morphological characteristics. J. Forensic Sci. 58, 53-58 (2013).

91. Andrade, R. N. M., Vieira, W. A., Bernardino, I. M., Franco, A. \& Paranhos, L. R. P. Reliability of palatal rugoscopy for sexual dimorphism in forensic dentistry: A systematic literature review and meta-analysis. Arch. Oral Biol. 97, 25-34 (2019).

92. Venkatesh, R. \& David, M. P. Cheiloscopy: An aid for personal identification. J. Forensic Dent. Sci. 3, 67-70 (2011).

93. Kannan, S., Muthu, K., Muthusamy, S. \& Sidhu, P. Cheiloscopy-A vital tool crime in crime investigation. Int. J. Forensic Sci. Pathol. 3, 89-93 (2015).

94. Silva, R. F. et al. Positive identification of skeletal remains combining smile photographs and forensic anthropology-A case report. J. Forensic Res. 6, 1-3 (2015).

95. Djuric, M., Rakocevic, Z. \& Djonic, D. The reliability of sex determination of skeletons from forensic context in the Balkans. Forensic Sci. Int. 147, 159-164 (2005).

\section{Author contributions}

A.F., L.K.G.L, M.N.O. and L.R.P. conceived the idea and had full roles in the identification, article review, data extraction, quality assessment, analysis, draft writing and revision of the manuscript. W.A.V., M.G.C. and C.B. took major roles in the analysis, manuscript draft preparation and revision. All authors read and approved the final version of the manuscript to be considered for publication. All authors also agreed to be equally accountable for all aspects of this research work.

\section{Funding}

This study was financed in part by CAPES-Finance Code 001. We are also thankful for the support of CNPq (Council for Scientific and Technological Development-Brazil)_Finance Code 307808/2018-1.

\section{Competing interests}

The authors declare no competing interests.

\section{Additional information}

Supplementary Information The online version contains supplementary material available at https://doi.org/ 10.1038/s41598-021-03680-3.

Correspondence and requests for materials should be addressed to L.R.P.

Reprints and permissions information is available at www.nature.com/reprints.

Publisher's note Springer Nature remains neutral with regard to jurisdictional claims in published maps and institutional affiliations.

Open Access This article is licensed under a Creative Commons Attribution 4.0 International License, which permits use, sharing, adaptation, distribution and reproduction in any medium or format, as long as you give appropriate credit to the original author(s) and the source, provide a link to the Creative Commons licence, and indicate if changes were made. The images or other third party material in this article are included in the article's Creative Commons licence, unless indicated otherwise in a credit line to the material. If material is not included in the article's Creative Commons licence and your intended use is not permitted by statutory regulation or exceeds the permitted use, you will need to obtain permission directly from the copyright holder. To view a copy of this licence, visit http://creativecommons.org/licenses/by/4.0/.

(c) The Author(s) 2021 